MITSUBISHI ELECTRIC RESEARCH LABORATORIES

http://www.merl.com

\title{
Random Access Protocols for Collaborative Spectrum Sensing in Multi-Band Cognitive Radio Networks
}

\author{
Chen, R-R.; Teo, K.H.; Farhang-Boroujeny.B.;
}

TR2011-044 February 2011

\begin{abstract}
In this paper, collaborative sensing and distributed detection are addressed in the context of multi-band cognitive radios. In a cognitive radio network, all the nodes may sense the spectrum simultaneously. They should then exchange their sensing results in order to improve the reliability of the detection. This exchange of information has to be done effectively to improve the bandwidth efficiency of the network. We propose a generalized medium access control (MAC) signaling protocol based on random access and study its performance through a thorough theoretical analysis. We begin with a nonadaptive protocol with fixed parameters. The numerical results obtained from analysis reveals that the fixed parameter protocol is not robust to the variation of the network conditions which, in general, are unknown a priori. We thus extend the proposed protocol to an adaptive one. Analysis of this adaptive protocol reveals its much superior performance. Our analysis covers a wide range of network conditions, including the case where some spectral activities may be hidden from a few of cognitive nodes and the case when a cognitive node senses only a subset of spectral bands. All theoretical results are corroborated through computer simulations.
\end{abstract}

IEEE Journal of Selected Topics in Signal Processing

This work may not be copied or reproduced in whole or in part for any commercial purpose. Permission to copy in whole or in part without payment of fee is granted for nonprofit educational and research purposes provided that all such whole or partial copies include the following: a notice that such copying is by permission of Mitsubishi Electric Research Laboratories, Inc.; an acknowledgment of the authors and individual contributions to the work; and all applicable portions of the copyright notice. Copying, reproduction, or republishing for any other purpose shall require a license with payment of fee to Mitsubishi Electric Research Laboratories, Inc. All rights reserved.

Copyright (C) Mitsubishi Electric Research Laboratories, Inc., 2011

201 Broadway, Cambridge, Massachusetts 02139 



\title{
Random Access Protocols for Collaborative Spectrum Sensing in Multi-Band Cognitive Radio Networks
}

\author{
Rong-Rong Chen, Koon Hoo Teo, and Behrouz Farhang-Boroujeny, Senior Member, IEEE
}

\begin{abstract}
In this paper, collaborative sensing and distributed detection are addressed in the context of multi-band cognitive radios. In a cognitive radio network, all the nodes may sense the spectrum simultaneously. They should then exchange their sensing results in order to improve the reliability of the detection. This exchange of information has to be done effectively to improve the bandwidth efficiency of the network. We propose a generalized medium access control (MAC) signaling protocol based on random access and study its performance through a thorough theoretical analysis. We begin with a nonadaptive protocol with fixed parameters. The numerical results obtained from analysis reveals that the fixed parameter protocol is not robust to the variation of the network conditions which, in general, are unknown $a$ priori. We thus extend the proposed protocol to an adaptive one. Analysis of this adaptive protocol reveals its much superior performance. Our analysis covers a wide range of network conditions, including the case where some spectral activities may be hidden from a few of cognitive nodes and the case when a cognitive node senses only a subset of spectral bands. All theoretical results are corroborated through computer simulations.
\end{abstract}

Index Terms-Cognitive radio (CR) networks, collaborative spectrum sensing, medium access control (MAC), multi-band, random access.

\section{INTRODUCTION}

A typical cognitive radio (CR) network [1] consists of a set of secondary users (SUs) that should coexist with primary users (PUs) of a shared broadband spectrum. PUs have a priority access to the spectrum over SUs. To utilize the spectrum holes (the portions of spectrum that are unused by PUs at a given time), SU network should be designed to aggregate more of the available bandwidth subject to minimum interference with the PUs. The hidden terminal problem [2], also, should be addressed to minimize the interference. For this purpose, the SU nodes should collaboratively sense the spectrum and decide which part of the spectrum is available to them. Accordingly, in a cognitive

Manuscript received November 13, 2009; revised March 28, 2010; accepted May 31,2010. Date of publication June 14, 2010; date of current version January 19,2011 . This work was supported in part by the National Science Foundation under Grants ECS-0547433 and ECS-0524720, and in part by Mitsubishi Electric Research Laboratories. The associate editor coordinating the review of this manuscript and approving it for publication was Vincent K. N. Lau.

R.-R. Chen and B. Farhang-Boroujeny are with the Department of Electrical and Computer Engineering, University of Utah, Salt Lake City, UT 84112 USA (e-mail: rchen@ece.utah.edu; farhang@ece.utah.edu).

K. H. Teo is with the Mitsubishi Electric Research Laboratories (MERL), Cambridge, MA 02421 USA (e-mail: teo@merl.com).

Color versions of one or more of the figures in this paper are available online at http://ieeexplore.iee.org.

Digital Object Identifier 10.1109/JSTSP.2010.2053017 network, communication may be established in a three-phase cognitive cycle. In the first cycle, all the cognitive nodes remain silent and listen to the spectral activities. This phase is called sensing. In the second phase, the information obtained by all the nodes is exchanged among them to improve the PU detection reliability of the SU network. We refer to this phase as signaling. Data transmission then follows over the remaining time of the cognitive cycle.

One may note that the duration of each cognitive cycle should be kept small to avoid significant interference with PUs. This is because, after each sensing phase, any new PU activities will be invisible to the SU network. Hence, to avoid a prolonged interference with PUs, the SU network should frequently update the available bands. Consequently, for a fixed length of cognitive cycle, to allow maximum transmission of data, the durations of sensing and signaling phases should be minimized. Efficient sensing methods have been proposed and widely studied in the literature; e.g., [1], [3]. The goal of this paper is to develop an effective protocol/strategy for minimizing the duration of the signaling phase.

To assure reliable collaborative sensing, in this paper, we assume signaling is established through a narrowband dedicated control channel (DCC). The DCC is a leased non-cognitive narrowband channel that is used for exchange of sensing information among SUs. We argue that this is a small price which one would like to pay for achieving reliable data communication in an environment with highly dynamic spectral activities.

From the above discussion, it is obvious that signaling overhead plays a major role in CR networks and thus should be given a due attention in any design. Nevertheless, so far very limited studies have been performed in this area. To overcome the hidden node problem, Wiess et al. [4] have proposed a boosting protocol where all the nodes in the network broadcast strong signals (i.e., shout) over the bands where they have observed PU activities thus reducing the need for a DCC. They argue, if boosting is done over a short period of time and only for newly allocated subbands, it incurs insignificant interference to PUs and thus may be acceptable. However, in many situations this violates noninterference requirements of PU network. Transform domain communication system and conventional contention scheme is proposed in [5] for access signaling of a network with a base station. Visotsky, et al. [6] analyze the probabilistic approach for collaborative detection under soft and hard information combining strategies. None of these works, however, considers the joint problem of detection and signaling to compute the overhead associated with spectrum exchange mechanism. Su and Zhang [7] study an analytical model of cognitive radio medium access control 
(MAC) with two types of channel sensing. The access mechanism, however, requires strong synchronization on mini-slot time scale. Although the time-slotted random access protocols considered in this paper also require user synchronization, it is possible to extend such protocols to unslotted ones, as is done for unslotted Aloha [8], to further reduce the synchronization requirement. The analysis for the un-slotted protocols, however, will be more involved due to the asynchronous nature of the protocol. Hence, in this work we focus on theoretical study of the more tractable time-slotted protocols. In [9], it is proposed that to minimize the interference with PUs, a DCC should be used for signaling. The signaling overhead is then studied through computer simulations. The use of a DCC have also been brought up in [10]-[13]. An interesting outcome of the presence of unreliable SUs in cooperative sensing is discussed in [11]. Further related works can be found in [14]-[20].

This paper extends our earlier work [21] where we proposed a random access protocol for collaborative exchange of sensing information using a DCC. In [21], we assumed that only a single-band channel was available for communications among SUs. The simplistic assumption that the probability of detection is the same for all SUs was also made. This work generalizes the contributions of [21] in a number of ways:

- The single-band network is extended to a multi-band communication network. We assume that a broadband channel is divided into a number of narrow PU bands and the proposed MAC protocol determines which PU bands are available to SUs.

- We develop a general mathematical framework that allows analysis of the proposed protocols under very broad conditions. In particular, our analysis allows consideration of hidden nodes and the cases where each node senses only a subset of PU bands.

- The protocol proposed in [21], for a single-band case, was nonadaptive, i.e., the protocol parameters were optimized and set fixed a priori. In this paper, we show that although this protocol behaves robustly in a single band network, in the sense that it is relatively insensitive to variation of the network parameters, it behaves poorly in a multi-band network, when the MAC/signaling protocol parameters are slightly varied around their optimal settings. We thus propose a modification to the proposed signaling protocol to make it adaptive. Theoretical results show that this adaptive protocol is far superior to the nonadaptive protocol and can be trusted for running networks with multiple PU bands.

Random access protocols have been widely used in communication networks where multiple users contend for channel access to maximize system throughput. In this paper, we propose to utilize random access protocols for a totally different objective- the broadcast and exchange of spectrum sensing information in a cognitive radio network. A distinctive feature of this design is that each SU in the network uses random access to broadcast his sensing information, and to update his own sensing information upon receiving a broadcast message from other SUs. In this sense, the random access protocols studied here are designed to allow efficient exchange of sensing information and to minimize the signaling period of a cognitive radio network. Furthermore, we develop theoretical analysis to analyze the performance of such protocols. To the best of our knowledge, this is the first work that thoroughly analyzes the random access protocols for collaborative spectrum sensing in cognitive radio networks.

In order to minimize the interference to the PUs, we assume that if one SU detects some PU activities over a PU band, then this information will be broadcast to all the SUs in the network and the SUs will refrain from accessing this PU band. It is possible that one SU could falsely detect the presence of a PU when it is actually absent, i.e., a false alarm occurs, and the false alarm will also be broadcast to other SUs, causing a degradation of the spectrum utilization of the SU network. This assumption, even though conservative, ensures high detection probabilities of the PUs and hence provide strong protection to the PU network. We also note that the random access protocols presented in this paper can be further generalized beyond the presentations in this paper. For instance, the adaptive protocol introduced in Section II-B considers only decreasing the parameter $\tau$, when the signaling traffic load is identified to be high. Clearly, one can modify the protocol to also increase $\tau$ when the signaling traffic load is identified to be low. We have chosen to limit the presentation in this paper to simpler protocols, because of the difficulty of the analysis. Even analysis of these simplified protocols has been a great challenge, as one may find out after reading the rest of this paper. Nevertheless, we believe that the simplifications are justifiable, as they allow us to develop a more in-depth understanding of random access protocols when applied to cooperative sensing. It is also worth noting that this analysis involves the definition and use of a number of events. To make the definitions more accessible to a reader that may need to refer to them as he reads the paper, all the event definitions are presented in text boxes throughout the paper.

The rest of the paper is organized as follows. Section II describes the basic system setup and the proposed protocols. In Section III, we describe the general sensing scenarios that our analysis of the random access protocols are applicable, including specific sensing examples. Analysis of the nonadaptive and adaptive protocols for general sensing scenarios are presented in Sections IV and V, respectively. Numerical results are given in Section VI. Finally, we conclude in Section VII.

\section{Cognitive Cycle AND Signaling Protocols}

Fig. 1 depicts a diagram that shows the three phases of the proposed cognitive cycle and the underlying signaling protocol. As discussed before, the three phases of the cognitive cycle are 1) sensing, during which all cognitive nodes remain silent and listen to the PUs' spectral activities (this is similar to the $s i$ lence period in IEEE802.22 [22]); 2) signaling, during which the SU nodes exchange their sensing information; and 3) data transmission, during which information are transmitted over the cognitive network.

During the signaling period, those SUs who have detected the PU activities will send broadcast messages (BMs) to other SUs, to improve the detection results for the whole network. The BMs are transmitted randomly in synchronized time slots, i.e., in a random access slotted ALOHA, [2]. For a BM to be successful during a time slot, only one SU can transmit during that time slot. For simplicity, we consider a single hop network, 


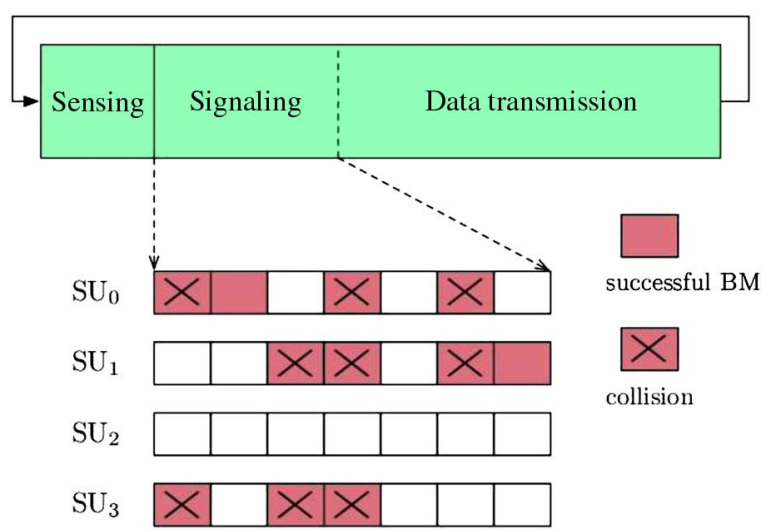

Fig. 1. Three phases of the proposed cognitive cycle and underlying signaling protocol.

where all the SUs are in close proximity to each other, (i.e., are in a single cell). Therefore, we assume that a successful BM can be heard by all SUs in the network, provided that suitable coding and modulation schemes are used to ensure the reliability of the BM. In general, since the PUs are located in a wider geographical region which might be hidden from a particular SU, collaborative spectrum sensing between the SUs is necessary to improve the detection probability of the PU activities.

In Fig. 1, we provide a simple example to illustrate the basic system setup, where $\mathrm{SU}_{0}$ and $\mathrm{SU}_{1}$ have successful BMs in the second and last time slots, respectively. If an SU fails to detect a PU locally, but receives a BM from other SUs saying that a PU has been detected, it will become aware of the existence of that PU in the respective PU band. Clearly, the detection probability of an SU will be improved after the signaling period. After signaling, data transmission begins over the available PU bands and continues for the rest of the cognitive cycle. The remaining parts of this paper concentrate on the signaling phase and develop analytical results which enable us to explore the random access behavior of both nonadaptive and adaptive protocols that are introduced next.

\section{A. Nonadaptive Protocol}

We first propose a nonadaptive protocol which realizes collaborative sensing through a $\tau$-persistent slotted ALOHA protocol. We first introduce some terminologies. We say that a PU band is busy if the PU band is being used by some PU. By the end of the sensing period, we say that an $\mathrm{SU}$ is active, if the SU detects at least one busy PU band. Each SU maintains a list of busy PU bands (LBB). After the sensing period, each SU's LBB contains only the busy PU bands that this SU has detected. Starting with the first time slot after the sensing period, each $a c$ tive $\mathrm{SU}$ attempts to transmit a BM, containing his LBB, through the control channel with a fixed probability of $\tau$. With probability of $1-\tau$, an active $\mathrm{SU}$ remains silent and listens to the control channel for a possible BM from other SUs. When multiple SUs attempt to transmit BM in the same time slot, a collision occurs. When only one SU attempts to transmit a BM, then we assume that the BM is received by every SU in the network and we refer to this BM as a successful BM (SBM). Upon receiving an SBM, each SU updates his LBB by including the new busy PU bands reported by the SBM. An SU will become inactive and stops transmitting, once he receives an SBM that contains all the busy PU bands in his own LBB. Since every $\mathrm{SU}$ in the network becomes aware of the LBB in the SBM, only those SUs whose LBBs contain additional busy PU bands will continue transmitting. An inactive user differs from an active user only in that he does not transmit any BM, and he still listens to the BM and updates his LBB according to the SBM.

We note that immediately after an SU transmits an SBM, he is not aware that the BM was transmitted successfully and thus will continue to transmit, i.e., remain active. We refer to this user as a dummy user because further transmissions of his BM do not provide new information about the PU activities, and only increase the possibility of BM collisions. The dummy user will become inactive, and thus no longer called a dummy user, when he receives an SBM from other SUs that includes his LBB. At which point, the user who transmitted the SBM will become the new dummy user. Clearly, the existence of the dummy user complicates analysis of the protocols and, thus, has to be given a due attention (see Sections IV and V).

\section{B. Adaptive Protocol}

As opposed to the nonadaptive protocol, we allow the SUs to adjust the transmission probability $\tau$ in time. Specifically, we implement the "multiplicative decrease" policy to let the SUs scale down their transmission probability when a collision occurs or after a transmission. The details are summarized as follows.

- After the sensing period, all the active SUs set their initial transmission probability to $\tau=\tau_{0}$.

- If the transmission probability of an SU is $\tau$ during a time slot and he attempts a transmission during this time slot, then his transmission probability during the next time slot will be scaled down to $\tau \cdot \alpha$.

- If a collision occurs during a time slot, then we assume that the collision will be detected by all the SUs who were not transmitting during this time slot. Each of these SUs will scale down his transmission probability for the next time slot to $\tau \cdot \alpha$.

- If an SU finds the DCC to be silent during one time slot, then he will not decrease his transmission probability.

- After each SBM, all the SUs (except for the SU who sent the SBM) will reset their transmission probability to $\tau=\tau_{0}$.

It is clear that the nonadaptive protocol is a special case of the adaptive protocol with a fixed transmission probability $\tau_{0}$ and a constant scaling factor $\alpha=1$. For the remainder of the paper, we use $\tau_{0}$ as the initial transmission probability for both protocols with the understanding that $\alpha=1$ for the nonadaptive protocol.

We note that for both the nonadaptive and adaptive protocols, the length of the signaling period is a parameter that is fixed a priori. An SU will remain active until he receives an SBM whose LBB contains his own LBB, or by the end of the signaling period. Next, we discuss how the length of the signaling period is determined. 


\section{Optimization of Length of the Signaling Period}

To maximize the bandwidth efficiency of the network, we let the length of the signaling period equals the minimum number of time slots after which all SUs are aware of all busy PU bands with a high probability $\eta$. To this end, let us define $P_{D}\left(n, \tau_{0}, \alpha\right)$ as the probability of all SUs being aware of all busy PU bands after $n$ time slots, given protocol parameters $\tau_{0}$ and $\alpha$. Then, the length of the signaling period can be set as

$$
N_{s}\left(\tau_{0}, \alpha\right)=\min \left\{n: P_{D}\left(n, \tau_{0}, \alpha\right) \geq \eta\right\}
$$

Clearly, $N_{s}\left(\tau_{0}, \alpha\right)$ can be large, for either large values of $\tau_{0}$, which leads to a significant number of BM collisions, or for small values of $\tau_{0}$, which leads to insufficient transmissions of BMs. In the analysis developed in this paper, we derive analytical expressions for computing $P_{D}\left(n, \tau_{0}, \alpha\right)$ and from which we can find the optimal $\tau_{\text {opt }}$ and $\alpha_{\text {opt }}$ to minimize the length of the signaling period as

$$
N_{\mathrm{opt}}=\min _{\tau_{0}, \alpha} N_{s}\left(\tau_{0}, \alpha\right)=N_{s}\left(\tau_{\mathrm{opt}}, \alpha_{\mathrm{opt}}\right)
$$

Note that (1), and hence, the optimization step (2), require perfect knowledge of the systems parameters such as the number of PU bands, the number of SUs, the PU band usage, and the sensing capability at each SU. In a realistic scenario, some of these parameters may be unknown, or can only be coarsely estimated. We demonstrate through numerical results in Section VI that the adaptive protocol is more robust than the nonadaptive protocol, in the sense that the length of the signaling period $N_{s}\left(\tau_{0}, \alpha\right)$ is less sensitive to $\tau_{0}, \alpha$, and other system parameters.

\section{SENSING SCENARIOS}

Performance of the proposed protocols clearly depend on the outcomes of the sensing period. In this section, we describe the general sensing scenarios for which the analysis developed in Sections IV and $\mathrm{V}$ are applicable. Here we assume that there are a total of $C$ (busy and idle) PU bands, denoted by $\left\{t_{1}, t_{2}, \cdots, t_{C}\right\}$. Let $c$ denote the number of busy PU bands, which can vary over cognitive cycles. To facilitate analysis, we make the symmetric assumption that statistically any group of $c_{1}$ out of $c$ busy PU bands have the same probability of being detected as any other groups of $c_{1}$ busy PU bands. In other words, we assume that the PU bands are statistically equivalent to each other. Without loss of generality, assume that the $c$ busy PU bands are $\left\{t_{1}, t_{2}, \cdots, t_{c}\right\}$. We also note that the analysis in Sections IV and V involves a conditioning step that depends on the number of busy PU bands contained in the first SBM (SBM1), and the number of remaining active users after receiving SBM1. To facilitate the computation of these probabilities, we introduce the following two sensing dependent events:

$$
\begin{array}{r}
X_{c_{1}, c}=\left\{\text { An SU detects }\left\{t_{1}, t_{2}, \cdots, t_{c_{1}}\right\}\right. \\
\text { as busy PU bands }\} \\
Y_{c_{1}}=\{\text { An SU does not detect any of the busy } \\
\text { PU bands in } \left.\left\{t_{1}, t_{2}, \cdots, t_{c_{1}}\right\}\right\}
\end{array}
$$

Due to the symmetry assumption, we see that $\left(\begin{array}{c}c \\ c_{1}\end{array}\right) P\left(X_{c_{1}, c}\right)$ determines the probability that SBM1 contains exactly $c_{1}$ busy PU bands. Here, the term $\left(\begin{array}{c}c \\ c\end{array}\right)$ takes care of the ordering of the PU bands. Also, given that SBM1 contains $c_{1}$ busy PU bands, an SU will remain active after receiving SBM1 if he detects at least one of the $c-c_{1}$ busy PU bands that are not included in SBM1. Again we apply the symmetry assumption to see that the probability of this event is determined by $1-P\left(Y_{c-c_{1}}\right)$. Note that whether an SU detects any of the $c_{1}$ PU bands already included in the SBM1 is irrelevant to whether he will remain active. This, combined with the symmetry assumption, lead to the term $P\left(Y_{c-c_{1}}\right)$. Detailed usage of $P\left(X_{c_{1}, c}\right)$ and $P\left(Y_{c_{1}}\right)$ can be found in Proposition 4.2.

Next, we consider two examples of sensing scenarios in Sections III-A and III-B, followed by a discussion of a general sensing scenario in Section III-C. For all cases analytical expressions of $P\left(X_{c_{1}, c}\right)$ and $P\left(Y_{c_{1}}\right)$ are developed.

\section{A. Full-Band Homogeneous Sensing}

Since the SUs are randomly located, it is reasonable to assume that they have different detection probabilities. We introduce a probability distribution $\left\{r_{1}, r_{2}, \cdots, r_{d}\right\}$, where $\sum_{i=1}^{d} r_{i}=1$ and a set of detection probabilities $\left\{q_{1}, \cdots, q_{d}\right\}$, where $0 \leq$ $q_{i} \leq 1$ for each $1 \leq i \leq d$. We assume that with probability $r_{i}$ an SU has a detection probability of $q_{i}$ for all of the busy PU bands. A small detection probability $q_{i}$ corresponds to a user who is at a location that PUs are hidden to him. For this scenario, one finds that

$$
P\left(X_{c_{1}, c}\right)=\sum_{i=1}^{d} r_{i} q_{i}^{c_{1}}\left(1-q_{i}\right)^{c-c_{1}}
$$

and

$$
P\left(Y_{c_{1}}\right)=\sum_{i=1}^{d} r_{i}\left(1-q_{i}\right)^{c_{1}}
$$

\section{B. Partial-Band Homogeneous Sensing}

Assume that each user randomly selects a total of $B$ PU bands to sense, given a total of $C$ (busy and silent) PU bands. As in the full-band sensing, the detection probability of a user is $q_{i}$ with probability $r_{i}$. Once $q_{i}$ is determined, each of the busy PU bands that is sensed by this user will be detected with probability $q_{i}$. It can be shown that, in this case, one obtains the results in (5) and (6). These results are proved in Appendix A:

$$
\begin{aligned}
P\left(X_{c_{1}, c}\right)=\frac{1}{\left(\begin{array}{l}
C \\
B
\end{array}\right)} \sum_{i=1}^{d} r_{i} \sum_{j=\max \left(c_{1}, B-(C-c)\right)}^{\min (B, c)}\left(\begin{array}{l}
c-c_{1} \\
j-c_{1}
\end{array}\right) \\
\times\left(\begin{array}{l}
C-c \\
B-j
\end{array}\right) q_{i}^{c_{1}}\left(1-q_{i}\right)^{j-c_{1}}
\end{aligned}
$$

for $1 \leq c_{1} \leq \min (B, c)$, and

$$
\begin{array}{r}
P\left(Y_{c_{1}}\right)=\frac{1}{\left(\begin{array}{l}
C \\
B
\end{array}\right)} \sum_{i=1}^{d} r_{i} \sum_{j=\max \left(0, B-\left(C-c_{1}\right)\right)}^{\min \left(B, c_{1}\right)}\left(\begin{array}{c}
c_{1} \\
j
\end{array}\right) \\
\times\left(\begin{array}{c}
C-c_{1} \\
B-j
\end{array}\right)\left(1-q_{i}\right)^{j}
\end{array}
$$

for $1 \leq c_{1} \leq c$. 


\section{General Sensing}

Next, we discuss a very general sensing scenario and show how $P\left(X_{c_{1}, c}\right)$ and $P\left(Y_{c}\right)$ can be computed for such scenarios as well.

Assume that there are a total of $C$ PU bands. There are a total of $T=2^{C}$ sensing outcomes, denoted by $\mathbf{u}_{1}, \cdots, \mathbf{u}_{T}$. Here, each sensing outcome $\mathbf{u}_{i}$ is a $C$-dimensional binary vector whose $j$ th component $u_{i j}$ equals one if the $j$ th PU band is detected busy. We let a $T$-dimensional vector $\mathbf{d}=\left(d_{1}, d_{2}, \cdots, d_{T}\right)$ denote a sensing distribution, where $d_{i}$ is the probability that the sensing outcome equals $\mathbf{u}_{i}$. Assume that a user chooses a sensing distribution $\mathbf{d}$ from a set of $n_{s}$ sensing distributions $\left\{\mathbf{z}_{1}, \mathbf{z}_{2}, \cdots, \mathbf{z}_{n_{s}}\right\}$ according to a probability distribution $P\left(\mathbf{d}=\mathbf{z}_{i}\right)=\gamma_{i}$, where $\sum_{i=1}^{n_{s}} \gamma_{i}=1$. For a fixed $c$, where $1 \leq c \leq C$, it is reasonable to make the assumption that, after averaging over this set of sensing distributions, the joint detection probabilities of any group of $c$ PU bands are the same. In other words, all the PU bands should be equivalent statistically. To compute $P\left(X_{c_{1}, c}\right)$ and $P\left(Y_{c_{1}}\right)$, we need to average over sensing distributions. To compute $P\left(X_{c_{1}, c}\right)$, we let $S_{c_{1}, c}$ denote all the sensing outcomes such that only the first $c_{1}$ PU bands are detected out of the first $c$ PU bands. Then we have

$$
P\left(X_{c_{1}, c}\right)=\sum_{i=1}^{n_{s}} \gamma_{i} \sum_{j: \mathbf{u}_{j} \in S_{c_{1}, c}} z_{i j}
$$

where $z_{i j}$ is the probability that the sensing outcome equals $\mathbf{u}_{j}$ given the sensing distribution $\mathbf{z}_{i}$. Similarly, let $V_{c_{1}}$ denote all the sensing outcomes such that none of the first $c_{1}$ PU bands is detected. Then, we have

$$
P\left(Y_{c_{1}}\right)=\sum_{i=1}^{n_{s}} \gamma_{i} \sum_{j: \mathbf{u}_{j} \in V_{c_{1}}} z_{i j} .
$$

\section{ANALYSIS OF NONADAPTIVE PROTOCOL}

Assume that the broadband channel that is shared between the PUs and SUs is divided into $C$ PU bands. We assume that there are $K \mathrm{SU}$ nodes. In general, the number of busy PU bands, denoted by $c$, is time-varying and is unknown a priori. For ease of disposition, we first consider the case when $c$ is fixed. The case of variable $c$ then easily follows.

\section{A. Fixed Number of Busy PU Bands}

Now, assume that there are a total of $c$ busy PU bands. We define the events

$$
\begin{array}{r}
A_{c, m}=\{\text { All SUs are aware of all } c \text { busy PU bands } \\
\text { by the end of time slot } m\} \\
B_{c, n}=\{\text { All SUs become aware of all } c \text { busy } \\
\text { PU bands the first in time slot } n\}
\end{array}
$$

While our goal is to compute $P\left(A_{c, m}\right)$

$$
P\left(A_{c, m}\right)=\sum_{n=0}^{m} P\left(B_{c, n}\right)
$$

it is sufficient to compute $P\left(B_{c, n}\right)$.

In Proposition 4.1 and 4.3 below, we develop recursive relations for computing $P\left(B_{c, n}\right)$. This is achieved by conditioning upon the first successful broadcast message (SBM1). Let us assume that SBM1 occurs during time slot $n_{1}$, and it contains $c_{1}$ busy PU bands. Then event $B_{c, n}$ will occur if, after receiving SBM1, all the SUs become aware of the remaining $c-c_{1}$ busy PU bands for the first time, after an additional $n-n_{1}$ slots. The reduction in the number of busy PU bands, from $c$ to $c-c_{1}$, and in the number of time slots, from $n$ to $n-n_{1}$, is crucial to facilitate the recursive computation of $P\left(B_{c, n}\right)$. Furthermore, due to the use of random access protocols, it is important to know the number of active users who attempt to transmit BMs. In particular, we need to know the number of active users immediately after the sensing period, represented by $k$, and also the number of remaining active users after receiving the SBM1, represented by $k_{1}$. These motivate us to define the following events:

$$
\begin{aligned}
& D_{k}=\{\text { There are } k \text { active users }\} \\
& \tilde{D}_{k}=\{\text { There are } k \text { (regular) active users } \\
& \text { and one dummy user }\} \\
& F_{n_{1}}=\left\{\mathrm{SBM} 1 \text { is transmitted in time slot } n_{1}\right\} \\
& G_{c_{1}, c}=\left\{\mathrm{SBM} 1 \text { reports } c_{1} \text { out of } c \text { busy PU bands }\right\} \\
& H_{k_{1}}=\left\{\text { After SBM } 1, k_{1} \text { SUs remain active }\right\} \\
& Q_{c_{1}, c}=\{\text { An active SU remains active after receiving } \\
& \text { SBM1 that reports } \left.c_{1} \text { out of } c \text { busy PU bands }\right\} \\
& U=\{\text { At least one SU does not detect all } c \text { busy } \\
& \text { PU bands by the end of the sensing period }\}
\end{aligned}
$$

\section{Proposition 4.1:}

$$
\begin{aligned}
P\left(B_{c, n}\right)= & \sum_{k=1}^{K} P\left(D_{k}\right) \sum_{n_{1}=1}^{n-1} P\left(F_{n_{1}} \mid D_{k}\right) P\left(B_{c, n} \mid D_{k}, F_{n_{1}}\right) \\
& +\sum_{k=1}^{K-1} P\left(D_{k}\right) P\left(F_{n} \mid D_{k}\right) P\left(G_{c, c}\right)+P\left(F_{n} \mid D_{K}\right) \\
& \times\left[P\left(D_{K} U\right)-P\left(D_{K}\right)\left(1-P\left(G_{c, c}\right)\right)\right]
\end{aligned}
$$

Proof: See Appendix B.

As shown in Proposition 4.1, the computation of $P\left(B_{c, n}\right)$ can be simplified by conditioning upon $k$ and $n_{1}$. For the special case when SBM1 occurs during slot $n$, i.e., $n_{1}=n$, we note that SBM1 must contain all busy PU bands, i.e., the event $G_{c, c}$ occurs. This relates to the last two terms in (10). For $n_{1}<n$, corresponding to the first term in (10), it is nontrivial to compute $P\left(B_{c, n} \mid D_{k}, F_{n_{1}}\right)$ directly, which will be handled separately in Proposition 4.3. For the nonadaptive protocol, one finds that

$$
P\left(F_{n_{1}} \mid D_{k}\right)=[1-\lambda]^{n_{1}-1} \lambda
$$

where $\lambda=k\left(1-\tau_{0}\right)^{k-1} \tau_{0}$ is the probability that only one out of $k$ SUs transmits during an arbitrary time slot. We note that the other events involved in Proposition 4.1, $D_{k}, D_{k} U, G_{c_{1}, c}$, and $Q_{c_{1}, c}$ are all sensing dependent and their probabilities can be calculated in terms of $P\left(X_{c_{1}, c}\right)$ and $P\left(Y_{c}\right)$ that were introduced in Section III. The following proposition gives the necessary formulas. 


\section{Proposition 4.2:}

$$
\begin{aligned}
P\left(D_{k}\right) & =\left(\begin{array}{c}
K \\
k
\end{array}\right)\left[1-P\left(Y_{c}\right)\right]^{k}\left[P\left(Y_{c}\right)\right]^{K-k} \\
P\left(D_{K} U\right) & =\left[1-P\left(Y_{c}\right)\right]^{K}-P\left(X_{c, c}\right)^{K} \\
P\left(G_{c_{1}, c}\right) & =\frac{\left(\begin{array}{c}
c \\
c_{1}
\end{array}\right) P\left(X_{c_{1}, c}\right)}{1-P\left(Y_{c}\right)} . \\
\left(Q_{c_{1}, c}\right) & =\frac{1-P\left(Y_{c-c_{1}}\right)}{1-P\left(Y_{c}\right)} .
\end{aligned}
$$

Proof: See Appendix D.

Proposition 4.3, shown next, presents an iterative procedure for computing $P\left(B_{c, n} \mid D_{k}, F_{n_{1}}\right)$.

Proposition 4.3: To compute $P\left(B_{c, n} \mid D_{k}, F_{n_{1}}\right)$ for $1 \leq k \leq$ $K$ and $1 \leq n_{1} \leq n-1$, iterate between the following recursive equations, starting with the initial value $P\left(B_{c, 1} \mid \tilde{D}_{k}\right)=$ $P\left(F_{1} \mid \tilde{D}_{k}\right) P\left(G_{c, c}\right)$ :

$$
\begin{aligned}
& P\left(B_{c, n} \mid D_{k}, F_{n_{1}}\right) \\
& =\sum_{c_{1}=1}^{c-1} \sum_{k_{1}=1}^{k-1} P\left(G_{c_{1}, c}\right) P\left(H_{k_{1}} \mid D_{k}, G_{c_{1}, c}\right) \\
& \quad \times P\left(B_{c-c_{1}, n-n_{1}} \mid \tilde{D}_{k_{1}}\right) \\
& P\left(B_{c, n} \mid \tilde{D}_{k}\right) \\
& =\frac{1}{k+1} \sum_{n_{1}=1}^{n-1} P\left(F_{n_{1}} \mid \tilde{D}_{k}\right) P\left(B_{c, n-n_{1}} \mid \tilde{D}_{k}\right) \\
& \quad+\frac{k}{k+1}\left[\sum_{n_{1}=1}^{n-1} P\left(F_{n_{1}} \mid \tilde{D}_{k}\right) P\left(B_{c, n} \mid D_{k}, F_{n_{1}}\right)\right] \\
& \quad+P\left(F_{n} \mid \tilde{D}_{k}\right) P\left(G_{c, c}\right) .
\end{aligned}
$$

Proof: See Appendix C.

In (16) of Proposition 4.3, a recursive relation is developed for computing $P\left(B_{c, n} \mid D_{k}, F_{n_{1}}\right)$, by conditioning upon the number of busy PU bands contained in SBM1, denoted by $c_{1}$, and the number of remaining active SUs after receiving SBM1, denoted by $k_{1}$. The term $P\left(G_{c_{1}, c}\right)$ is the probability that the SBM1 contains $c_{1}$ busy PU bands, and $P\left(H_{k_{1}} \mid D_{k}, G_{c_{1}, c}\right)$ is the probability that after receiving SBM1, $k_{1}$ active users will remain active to participate in the broadcast of the remaining $c-c_{1}$ busy PU bands. The last term $P\left(B_{c-c_{1}, n-n_{1}} \mid \tilde{D}_{k_{1}}\right)$ is the probability that given $k_{1}$ active users and one dummy user, the probability that all users will become aware of the remaining $c-c_{1}$ busy PU bands for the first time after $n-n_{1}$ slots. Note that conditioning upon SBM1 allows the reduction in the recursive relation in both the number of busy PU bands and the number of active users. The computation of $P\left(B_{c, n} \mid \tilde{D}_{k}\right)$ in (17) conditions upon whether a dummy user or a regular user succeeds in transmitting the next SBM. With probability $1 / k+1$, the dummy user succeeds and the SBM does not contain any new information to the network. This corresponds to the first term of (17). Otherwise, with probability $k / k+1$, a regular user succeeds and the SBM contains useful information to the network. This corresponds to the last two terms of (17).
To complete iterations between (16) and (17), one also needs to evaluate $P\left(F_{n_{1}} \mid \tilde{D}_{k}\right)$ and $P\left(H_{k_{1}} \mid D_{k}, G_{c_{1}, c}\right)$. It is straightforward to see that

$$
P\left(F_{n_{1}} \mid \tilde{D}_{k}\right)=P\left(F_{n_{1}} \mid D_{k+1}\right)
$$

and

$$
\begin{aligned}
& P\left(H_{k_{1}} \mid D_{k}, G_{c_{1}, c}\right)=\left(\begin{array}{c}
k-1 \\
k_{1}
\end{array}\right) {\left.\left[P\left(Q_{c_{1}, c}\right)\right]\right)^{k_{1}} } \\
& \times\left[1-P\left(Q_{c_{1}, c}\right)\right]^{k-1-k_{1}}
\end{aligned}
$$

where the term $P\left(Q_{c_{1}, c}\right)$ is computed using (15).

\section{B. Variable Number of Busy PU Bands}

In Section IV-A, we examined performance of the nonadaptive protocol for the case when the number of busy PU bands $c$ is fixed. Here we consider the case of variable number of busy PU bands. We assume that the collaborative sensing period is much shorter than the time scale of change in the PU band occupation such that the busy PU bands can be detected reliably. In other words, the PU activities do not change within a cognitive cycle, but can change from cycle to cycle. During one cognitive cycle, we assume that each PU band is busy with a probability of $0<p_{a}<1$. Hence, the number of busy PU bands $c$ varies from one cognitive cycle to another. For brevity, we let $P_{D}(n)=P_{D}\left(n, \tau_{0}, \alpha\right)$ denote the probability that all the SUs are aware of all the busy PU bands by time $n$. Since $c$ varies over cognitive cycles, we let $P(c)$ be the probability that there are $c$ busy PU bands during a cognitive cycle. Clearly, $P(c)$ follows a binomial distribution with parameters $C$ and $p_{a}$, here $C$ is the total number of PU bands. Thus, we obtain

$$
\begin{aligned}
P_{D}(n) & =\sum_{c=0}^{C} P\left(A_{c, n}\right) P(c) \\
& =\sum_{c=0}^{C} P\left(A_{c, n}\right)\left(\begin{array}{l}
C \\
c
\end{array}\right) p_{a}^{c}\left(1-p_{a}\right)^{C-c}
\end{aligned}
$$

where $P\left(A_{c, n}\right)$ can be computed via $P\left(B_{c, n}\right)$ using (9).

\section{AnAlysis of AdAPtive Protocol}

The main difference between the nonadaptive protocol and the adaptive protocol is that the latter allows the SUs to adaptively adjust the transmission probability $\tau$ in time. In particular, the transmission probability of the dummy user may differ from that of a regular active user. These make the analysis of the adaptive protocol more involved, even though it still follows similar approaches as that of the nonadaptive protocol. In order to keep track of the SUs' transmission probabilities under the adaptive protocol, it is necessary to generalize some of the previously defined events for the nonadaptive protocol. For instance, the event $F_{n_{1}}$ is now generalized to $F_{n_{1}, z_{1}}$, where $z_{1}$ is the number of collisions until SBM1. Clearly, $z_{1}$ determines the transmission probability of dummy user immediately after SBM1 is sent. For the same reason, the event $\tilde{D}_{k}$ is generalized to $\hat{D}_{k, z_{d}, z_{r}}$, which specifies not only the number of active users $k$, but also the transmission probabilities, i.e., $\tau_{r}=\tau_{0} \alpha^{z_{r}}$ for the regular 
user, and $\tau_{d}=\tau_{0} \alpha^{z_{d}}$ for the dummy user. These new events are defined as follows:

$F_{n_{1}, z_{1}}=\left\{\mathrm{SBM} 1\right.$ is transmitted in time slot $n_{1}$ after
$z_{1}$ collisions $\}$
$\hat{D}_{k_{1}, z_{d}, z_{r}}=\left\{\right.$ There are $k_{1}$ regular active users,
each with a transmission probability of
$\tau_{r}=\tau_{0} \alpha^{z_{r}}$, plus a dummy user with a
transmission probability of $\left.\tau_{d}=\tau_{0} \alpha^{z_{d}}\right\}$
$\hat{D}_{k_{1}, z_{d}}=\hat{D}_{k_{1}, z_{d}, 0}$
$E_{0}=\{$ No one transmits during time slot 1$\}$
$E_{c}=\{$ A collision occurs during time slot 1$\}$
$R=\{$ An SBM is transmitted by a dummy user $\}$

Given these newly defined events, we can generalize previous results for the nonadaptive protocol to the adaptive protocol. First, by replacing $F_{n_{1}}$ by $F_{n_{1}, z_{1}}$ and adding an additional summation over $z_{1}$, we generalize Proposition 4.1 to Proposition 5.1 presented next.

Proposition 5.1:

$$
\begin{aligned}
P\left(B_{c, n}\right)= & \sum_{k=1}^{K} P\left(D_{k}\right) \sum_{n_{1}=1}^{n-1} \sum_{z_{1}=0}^{n_{1}-1} P\left(F_{n_{1}, z_{1}} \mid D_{k}\right) \\
& \times P\left(B_{c, n} \mid D_{k}, F_{n_{1}, z_{1}}\right) \\
& +\sum_{k=1}^{K-1} P\left(D_{k}\right) \sum_{z_{1}=0}^{n_{1}-1} P\left(F_{n_{1}, z_{1}} \mid D_{k}\right) P\left(G_{c, c}\right) \\
& +\sum_{z_{1}=0}^{n-1} P\left(F_{n, z_{1}} \mid D_{K}\right) \\
& \times\left[P\left(D_{K} U\right)-P\left(D_{K}\right)\left(1-P\left(G_{c, c}\right)\right)\right]
\end{aligned}
$$

Proof: This follows from (10) once we write $F_{n_{1}}=$ $\bigcup_{z_{1}=0}^{n_{1}-1} F_{n_{1}, z_{1}}$.

Second, Proposition 4.3 can be generalized to Proposition 5.2 presented next.

Proposition 5.2:

$$
\begin{aligned}
P( & \left.B_{c, n} \mid D_{k}, F_{n_{1}, z_{1}}\right) \\
= & \sum_{c_{1}=1}^{c-1} \sum_{k_{1}=1}^{k-1} P\left(G_{c_{1}, c}\right) P\left(H_{k_{1}} \mid D_{k}, G_{c_{1}, c}\right) \\
& \quad \times P\left(B_{c-c_{1}, n-n_{1}} \mid \tilde{D}_{k_{1}, z_{1}+1}\right) \\
P( & \left.B_{c, n} \mid \hat{D}_{k, z_{d}}\right) \\
= & \sum_{n_{1}=1}^{n-1} \sum_{z_{1}=0}^{n_{1}-1} P\left(F_{n_{1}, z_{1}} \mid \hat{D}_{k, z_{d}}\right) P\left(R \mid \hat{D}_{k, z_{d}}, F_{n_{1}, z_{1}}\right) \\
& \times P\left(B_{c, n-n_{1}} \mid \hat{D}_{k, z_{d}+z_{1}+1}\right) \\
& +\sum_{n_{1}=1}^{n-1} \sum_{z_{1}=0}^{n_{1}-1} P\left(F_{n_{1}, z_{1}} \mid \hat{D}_{k, z_{d}}\right) P\left(\bar{R} \mid \hat{D}_{k, z}, F_{n_{1}, z_{1}}\right) \\
& \times P\left(B_{c, n} \mid D_{k}, F_{n_{1}, z_{1}}\right) \\
& +\sum_{z_{1}=0}^{n-1} P\left(F_{n, z_{1}} \mid \hat{D}_{k, z_{d}}\right) P\left(G_{c, c}\right)
\end{aligned}
$$

Proof: See Appendix E.
Compared to (17) for the nonadaptive protocol, we note that in (23), an additional summation over $z_{1}$ is required to track the transmission probability of SUs. Furthermore, given $k$ regular users and one dummy user in the system, the probability that the dummy user transmits the SBM is no longer $1 /(k+1)$, as is for the nonadaptive protocol. This is because the transmission probability of the dummy user can differ from that of the regular user. We take this into account in the term $P\left(R \mid \hat{D}_{k, z_{d}}, F_{n_{1}, z_{1}}\right)$, which is computed in (47).

Since $P\left(H_{k_{1}} \mid D_{k}, G_{c_{1}, c}\right)$ is sensing-dependent, (19) holds here as well. Moreover, the equations stated in Proposition 4.2 are also applicable to the adaptive protocol. This is because the events involved are protocol-independent. The computations of the protocol dependent events $P\left(F_{n_{1}, z_{1}} \mid D_{k}\right)$ and $P\left(F_{n_{1}, z_{1}} \mid \tilde{D}_{k, z}\right)$ in (21)-(23), however, are less straightforward. They may be computed recursively following Proposition 5.3.

Proposition 5.3: Starting with the initial value $P\left(F_{1,0} \mid \hat{D}_{k, z_{d}, z_{r}}\right)=1-P\left(E_{0} \mid \hat{D}_{k, z_{d}, z_{r}}\right)-P\left(E_{c} \mid \hat{D}_{k, z_{d}, z_{r}}\right)$, iterate, for $z_{1}=1,2, \cdots, n_{1}-1$

$$
\begin{aligned}
P( & \left.F_{n_{1}, z_{1}} \mid \hat{D}_{k, z_{d}, z_{r}}\right) \\
= & P\left(E_{0} \mid \hat{D}_{k, z_{d}, z_{r}}\right) P\left(F_{n_{1}, z_{1}} \mid \hat{D}_{k, z_{d}, z_{r}}, E_{0}\right) \\
& +P\left(E_{c} \mid \hat{D}_{k, z_{d}, z_{r}}\right) P\left(F_{n_{1}, z_{1}} \mid \hat{D}_{k, z_{d}, z_{r}}, E_{1}\right) \\
= & P\left(E_{0} \mid \hat{D}_{k, z_{d}, z_{r}}\right) P\left(F_{n_{1}-1, z_{1}} \mid \hat{D}_{k, z_{d}, z_{r}}\right) \\
& +P\left(E_{c} \mid \hat{D}_{k, z_{d}, z_{r}}\right) \\
& \times P\left(F_{n_{1}-1, z_{1}-1} \mid \hat{D}_{k, z_{d}+1, z_{r}+1}\right)
\end{aligned}
$$

where

$$
\begin{aligned}
P\left(E_{0} \mid \hat{D}_{k, z_{d}, z_{r}}\right)= & \left(1-\tau_{r}\right)^{k}\left(1-\tau_{d}\right) \\
P\left(E_{c} \mid \hat{D}_{k, z_{d}, z_{r}}\right)= & 1-\left(1-\tau_{r}\right)^{k}\left(1-\tau_{d}\right)-\left(1-\tau_{r}\right)^{k} \tau_{d} \\
& -k\left(1-\tau_{r}\right)^{k-1} \tau_{r}\left(1-\tau_{d}\right) \\
= & 1-\left(1-\tau_{r}\right)^{k-1} \\
& \times\left[1-\tau_{r}+k \tau_{r}\left(1-\tau_{d}\right)\right] .
\end{aligned}
$$

Once $P\left(F_{n_{1}, z_{1}} \mid \hat{D}_{k, z_{d}, z_{r}}\right)$ is computed, we have $P\left(F_{n_{1}, z_{1}} \mid \hat{D}_{k, z_{d}}\right)=P\left(F_{n_{1}, z_{1}} \mid \hat{D}_{k, z_{d}, 0}\right)$

$P\left(F_{n_{1}, z_{1}} \mid D_{k}\right)= \begin{cases}0, & \text { if } k=1, z_{1}>0 \\ \left(1-\tau_{0}\right)^{n_{1}-1} \tau_{0}, & \text { if } k=1, z_{1}=0 \\ P\left(F_{n_{1}, z_{1}} \mid \hat{D}_{k-1,0,0}\right), & \text { if } k \geq 2 .\end{cases}$

We note that the iterative relation given in (24) is obtained by conditioning upon whether a collision occurs in the first time slot. If no one transmits during this time slot, then the transmission probability of each SU remains the same, and SBM1 will occur after additional $n_{1}-1$ slots and $z_{1}$ collisions, corresponding to the term $P\left(F_{n_{1}-1, z_{1}} \mid \hat{D}_{k, z_{d}, z_{r}}\right)$ in (24). If a collision occurs during the first time slot, then each SU will scale down the transmission probability by $\alpha$, and SBM1 will occur after additional $n_{1}-1$ slots and $z_{1}-1$ collisions. This leads to the term $P\left(F_{n_{1}-1, z_{1}-1} \mid \hat{D}_{k, z_{d}+1, z_{r}+1}\right)$ in (24).

Finally, as in the case of the nonadaptive protocol, we first compute $P\left(B_{c, n}\right)$ using Proposition 5.2 for the case when the 


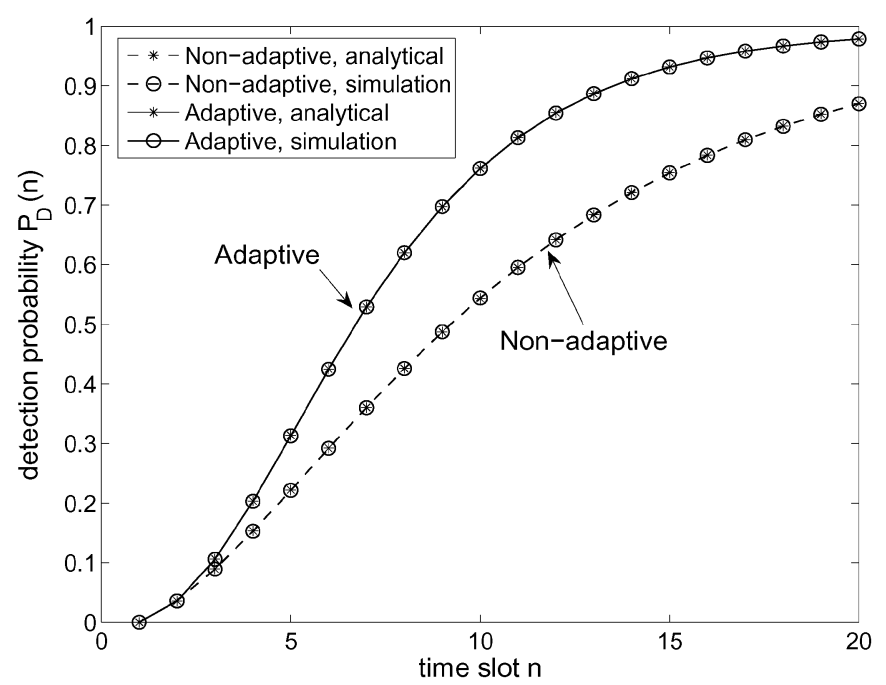

Fig. 2. Comparisons of simulation results and analytical expressions for fullband homogeneous sensing. The total number of PU bands is $C=6$, each PU band is busy with probability $p_{a}=0.8$. The total number of SU is $K=10$, each senses all the PU bands. The detection probabilities are determined by $\left[q_{1}, q_{2}\right]=[0.7,0.1],\left[r_{1}, r_{2}\right]=[0.65,0.35]$. The adaptive protocol assumes an initial $\tau_{0}=0.3$ and the scaling constant $\alpha=0.7$. The nonadaptive protocol uses a fixed $\tau_{0}=0.3$.

number of busy PU bands is fixed. Extension of the results to the variable case is straightforward since (20) is also applicable once $P\left(B_{c, n}\right)$, and thus $P\left(A_{c, n}\right)$ from (9), are computed.

\section{NUMERICAL RESULTS}

In this section, we provide numerical results to assess the performance of the proposed random access protocols. The numerical results also serve to verify the accuracy of the analysis developed in Sections IV and V through comparisons with actual simulations.

\section{A. Simulation Setup}

We adopt similar simulation setup for both full-band homogeneous sensing and partial-band homogeneous sensing. Assume that there are a total of $C$ PU bands (including busy and idle bands), and each band is busy with a probability of $p_{a}=0.8$. We run simulation for many independent cognitive cycles and assume that whether or not a PU band is busy is independent from cycle to cycle. Hence, the probability that there are $c$ busy PU bands in a typical cognitive cycle equals $P(c)=\left(\begin{array}{l}C \\ c\end{array}\right) p_{a}^{c}\left(1-p_{a}\right)^{C-c}$, where $c=0, \cdots, C$. Clearly, while $c$ remains the same within each cognitive cycle, it varies from cycle to cycle. The total number of SUs who participate in the collaborative sensing is $K$. Depending on the specific sensing scenario, each SU conducts either full-band sensing or partial-band sensing, and the sensing distribution is independent from user to user. After the sensing period, the SUs exchange their sensing information according to either nonadaptive, or adaptive protocol. For each time slot $n$, we check whether all the SUs become aware of all the busy PU bands for that particular cognitive cycle. We run many independent cognitive cycles and count the number of cognitive cycles during which all the SUs become aware of all the busy PU bands by time slot $n$. Then we divide it by the total number of cognitive cycles to obtain a simulated value for the detection probability $P_{D}(n)$, defined in (20).

\section{B. Full-Band Homogeneous Sensing}

We first consider the full-band homogeneous sensing scenario described in Section III-A. Here, we let $K=10, C=6$, and $p_{a}=0.8$. We consider $\left[q_{1}, q_{2}\right]=[0.7,0.1]$ and $\left[r_{1}, r_{2}\right]=$ $[0.65,0.35]$. This approximates a mobile scenario where an SU is moving inside a cell and with probability 0.35 it is at a location that is hidden from the PUs and therefore has a low detection probability of 0.1 . For each simulation run, we randomly generate a set of busy PU bands according to $p_{a}=0.8$, and each $\mathrm{SU}$ also randomly chooses his detection probability following the distribution of $\left[r_{1}, r_{2}\right]=[0.65,0.35]$. Based on the initial sensing outcome, the proposed random access protocols are employed to facilitate exchange of sensing information. Here we consider the nonadaptive protocol with $\tau_{0}=0.3$ and the adaptive protocol with $\tau_{0}=0.3$ and $\alpha=0.7$. Through extensive simulation consisting of $10^{6}$ simulation runs, we obtain $P_{D}(n)$ as a function of the number of time slots $n$. This is compared with the analytical value computed from (20). Fig. 2 demonstrates excellent match between the simulated value and the analytical value for both protocols. Even though for this particular protocol parameter $\tau_{0}=0.3$, the adaptive protocol yields a higher detection probability $P_{D}(n)$ than that of the nonadaptive protocol, the detection performance of these two protocols can be similar, provided that each uses its own optimized protocol parameters. Such optimization, however, becomes infeasible when some of the system parameters are unknown a priori. Hence, the adaptive protocol is more advantageous in that its performance is more robust to parameter variations.

Next, we study the performance of these two protocols as functions of protocol parameters. While the performance of the adaptive protocol depends on the scaling constant $\alpha$, we find that $\alpha=0.7$ seems to be a good value for all the test cases that we have examined. Hence, for simplicity, we keep $\alpha=0.7$ fixed for the adaptive protocol and study the protocol performance as a function of $\tau_{0}$. Let $N_{s}\left(\tau_{0}\right)$ denote the minimum number of time slots required such that $P_{D}(n) \geq \eta$, where $\eta=0.95$. In Fig. 3, we plot $N_{s}\left(\tau_{0}\right)$ versus $\tau_{0}$ for the two protocols and $N_{s}\left(\tau_{0}\right)$ is computed from the analytical formula (20). The system parameters are the same as those used in Fig. 2, with the exception that we consider another set of detection probabilities $\left[q_{1}, q_{2}\right]=[0.9,0.1]$. It is clear from Fig. 3 that, for the adaptive protocol, the optimal length of the signaling period, given by $N_{\text {opt }}=\min _{\tau_{0}} N_{s}\left(\tau_{0}\right)$, is approximately achieved over a wide range of $\tau_{0} \in[0.15,0.4]$. In comparison, the nonadaptive protocol is sensitive to $\tau_{0}$ and $N_{s}\left(\tau_{0}\right)$ increases rapidly when $\tau_{0} \in[0.25,0.4]$. Even though the $N_{\text {opt }}$ achieved by the optimized $\tau_{0}$ are similar for both protocols, the adaptive protocol is more advantageous in that $N_{\text {opt }}$ is achieved approximately over a wide range of $\tau_{0}$, thus the protocol is more robust with respect to system parameters such as detection probabilities and the number of users in the system. Another observation from Fig. 3 is that, when the detection probability $\left[q_{1}, q_{2}\right]$ is larger 


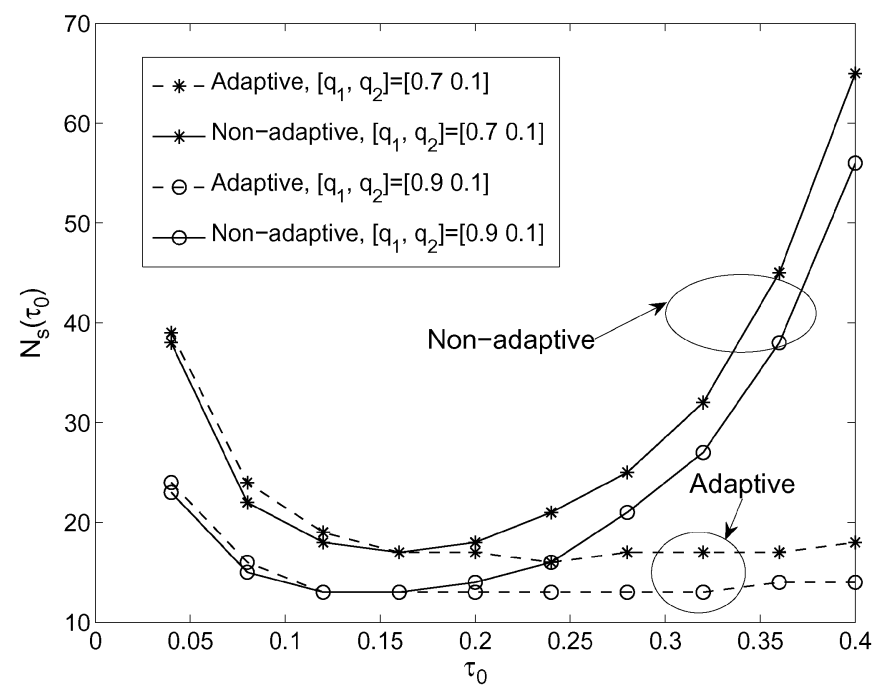

Fig. 3. Comparisons of nonadaptive and adaptive protocol for full-band homogeneous sensing. The total number of PU bands is $C=6$, each PU band is busy with probability $p_{a}=0.8$. The total number of SU is $K=10$, each senses all the PU bands. We consider two sets of parameters $\left[q_{1}, q_{2}\right]=[0.7,0.1]$, $\left[r_{1}, r_{2}\right]=[0.65,0.35]$ and $\left[q_{1}, q_{2}\right]=[0.9,0.1],\left[r_{1}, r_{2}\right]=[0.65,0.35]$. Here $N_{s}\left(\tau_{0}\right)$ denotes the minimum number of times slots required such that $P_{D}(n) \geq \eta=0.95$.

(i.e., the sensing quality of each SU is better), the network requires a smaller $N_{\text {opt }}$ to achieve the desired detection reliability.

\section{Partial-Band Homogeneous Sensing}

Next, we consider the partial-band homogeneous sensing scenario described in Section III-B with $K=10, C=6, p_{a}=0.8$. For each simulation run, every SU randomly senses $B=4 \mathrm{PU}$ bands. The detection probability of the SU is randomly generated according to $\left[q_{1}, q_{2}, q_{3}\right]=[0.8,0.7,0.6]$ and $\left[r_{1}, r_{2}, r_{3}\right]=$ $[0.3,0.55,0.15]$. As shown in Fig. 4 , with $\tau_{0}=0.2$, the analytical formula (20) matches with the simulation results precisely for both protocols.

For the same system setup, in Fig. 5 we examine $N_{s}\left(\tau_{0}\right)$ as a function of $\tau_{0}$ for two systems with $K=20$ and $K=10$, respectively. Similar to Fig. 3, the adaptive protocol is shown to be more robust than the nonadaptive protocol in that $N_{\text {opt }}$ is achieved approximately over a wide range of $\tau_{0}$. In comparison, for the nonadaptive protocol $N_{s}\left(\tau_{0}\right)$ increases rapidly for larger values of $\tau_{0}$. We note that the adaptive protocol is also more robust to $K$, as $N_{s}\left(\tau_{0}\right)$ remains close for $K=10$ and $K=20$ over a wide range of $\tau_{0}$. The nonadaptive protocol, on the other hand, is clearly sensitive to the values of $K$. Another observation from Fig. 5 is that, for the adaptive protocol, we have $\left(N_{\text {opt }}, \tau_{\text {opt }}\right) \approx(19,0.1)$ for $K=20$ and $\left(N_{\text {opt }}, \tau_{\text {opt }}\right) \approx(20,0.18)$ for $K=10$. Even though the optimal transmission probability $\tau_{\text {opt }}$ for $K=20$ is roughly half of that for $K=10$, we note that $N_{\text {opt }}$ is slightly smaller for $K=20$ than for $K=10$. This can be explained as follows. First, when more SUs are involved in collaborative sensing, it is more likely that some of the SUs will obtain good sensing results. Hence, collectively, the sensing outcome for $K=20$ should be better than that for $K=10$. Second, for $K=20$, since there are more active users who will attempt transmissions, it is necessary to reduce the transmission probability of each SU to pre-

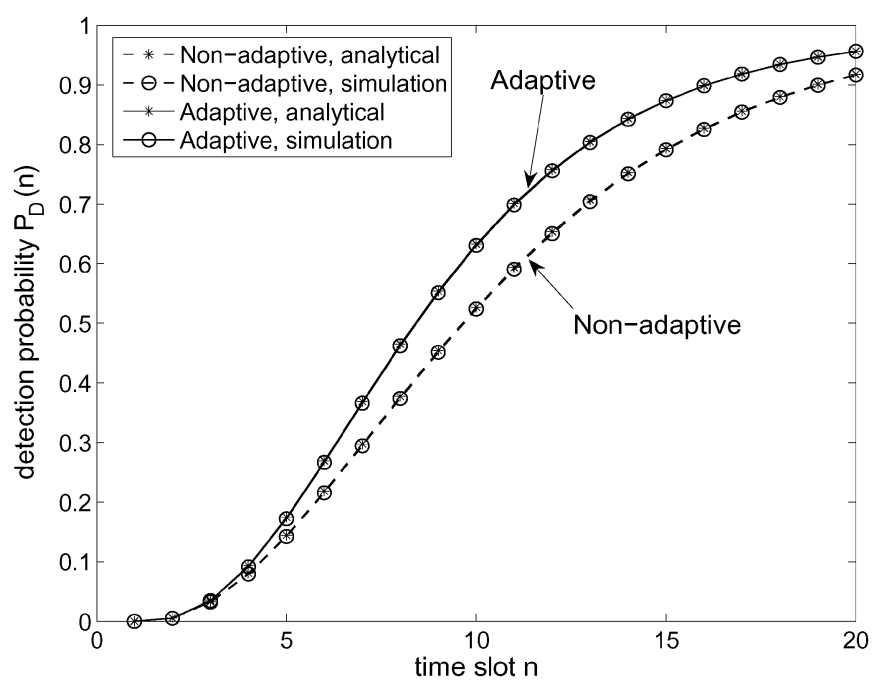

Fig. 4. Comparisons of simulation results and analytical expressions for partial-band homogeneous sensing. The total number of PU bands is $C=6$, and each is busy with a probability of $p_{a}=0.8$. Each SU randomly chooses $B=4$ PU bands to sense. The detection probabilities are determined by $\left[q_{1}, q_{2}, q_{3}\right]=$ $[0.8,0.7,0.6]$ and $\left[r_{1}, r_{2}, r_{3}\right]=[0.3,0.55,0.15]$. The adaptive protocol assumes an initial $\tau_{0}=0.2$ and the scaling constant $\alpha=0.7$. The nonadaptive protocol uses a fixed $\tau_{0}=0.2$.

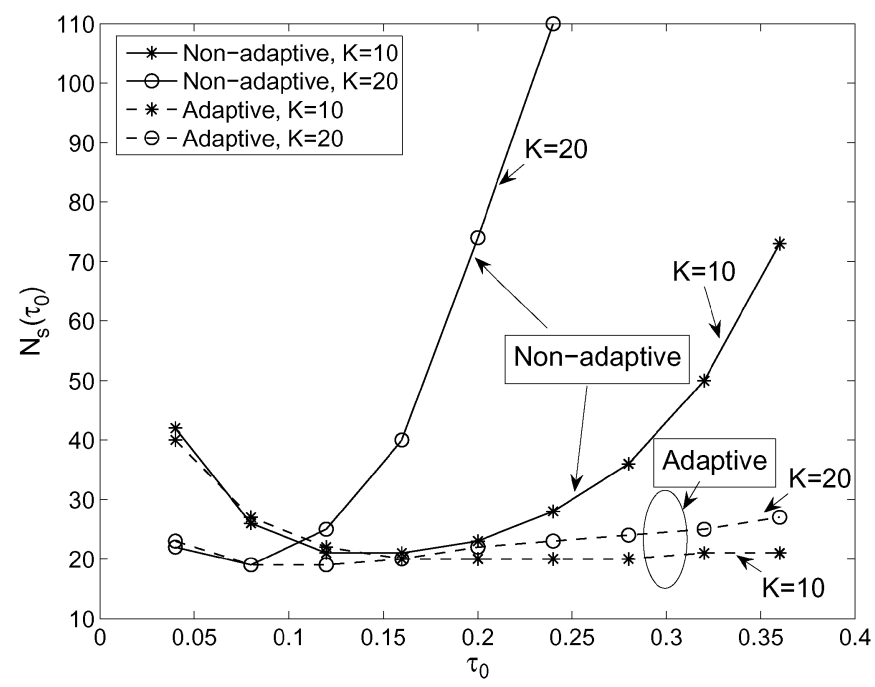

Fig. 5. Comparisons of nonadaptive and adaptive protocol for partial-band homogeneous sensing. The total number of PU bands is $C=6$, and each is busy with a probability of $p_{a}=0.8$. Each SU randomly chooses $B=4 \mathrm{PU}$ bands to sense. The detection probabilities are determined by $\left[q_{1}, q_{2}, q_{3}\right]=$ $[0.8,0.7,0.6]$ and $\left[r_{1}, r_{2}, r_{3}\right]=[0.3,0.55,0.15]$. Here $N_{s}\left(\tau_{0}\right)$ denotes the minimum number of times slots required such that $P_{D}(n) \geq \eta=0.95$.

vent too many collisions. Nevertheless, since there are more SUs for $K=20$, we still have sufficient transmissions of broadcast messages despite the reduction in transmission probability per SU. For these two reasons, we see that intuitively, it is reasonable that $N_{\text {opt }}$ is smaller for $K=20$ than for $K=10$, given better initial sensing outcomes and appropriate choice of transmission probabilities. Consequently, the advantage of larger $K$ will be more pronounced for cases where the detection probability of each SU is inferior.

In Fig. 6, we compare performance of full-band sensing $B=$ 6 with partial-band homogeneous sensing $B=4$ for both adaptive and nonadaptive protocols. Here we include two curves from Fig. 5 for partial-band sensing, and add two new curves 


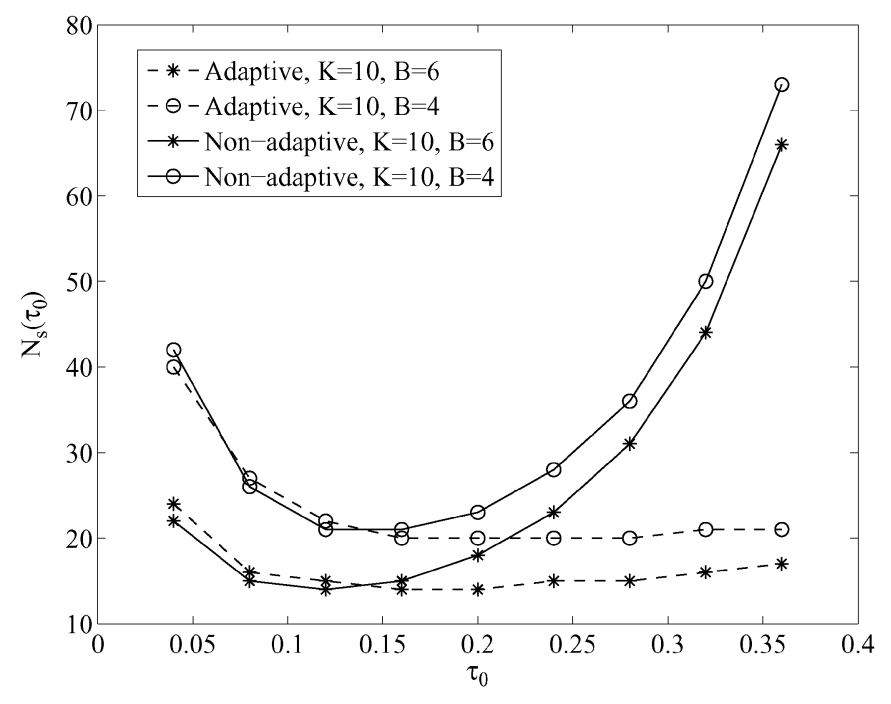

Fig. 6. Comparisons of full-band and partial-band homogeneous sensing. The total number of PU bands is $C=6$, and each is busy with a probability of $p_{a}=$ 0.8 . The detection probabilities are determined by $\left[q_{1}, q_{2}, q_{3}\right]=[0.8,0.7,0.6]$ and $\left[r_{1}, r_{2}, r_{3}\right]=[0.3,0.55,0.15]$. We let $B=4$ for partial-band sensing and $B=6$ for full-band sensing. Here $N_{s}\left(\tau_{0}\right)$ denotes the minimum number of times slots required such that $P_{D}(n) \geq \eta=0.95$.

for full-band sensing, using the same parameters $\left[q_{1}, q_{2}, q_{3}\right]=$ $[0.8,0.7,0.6]$ and $\left[r_{1}, r_{2}, r_{3}\right]=[0.3,0.55,0.15]$. It is clear from Fig. 6 that full-band sensing requires smaller $N_{s}\left(\tau_{0}\right)$ than that of partial-band sensing, because full-band sensing provides superior sensing outcomes after the sensing period, and hence fewer SBMs are needed to achieve the target detection probability. In general, partial-band sensing is advantageous in that it can reduce the amount of sensing resources required. This, however, is achieved at the cost of increased signaling overhead, as shown in Fig. 6.

\section{Selection of $\tau_{0}$}

Ideally, we want to choose the best $\tau_{\text {opt }}$ to minimize $N_{s}\left(\tau_{0}\right)$, which requires perfect knowledge of the system parameters including $K, C, p_{a}$ and the sensing distribution. If all $K$ SUs are active and $K$ is known perfectly, we note that $\tau_{0}=1 / K$ gives a good approximation of $\tau_{\text {opt }}$ because it minimizes the probability of collision for the first time slot of the signaling period. When $K$ is unknown, one should start with a rough estimate, preferably a larger value of $\tau_{0}$ to initialize the protocol. This is because when the initial $\tau_{0}$ is too small, too few BMs will be transmitted which will increase $N_{s}\left(\tau_{0}\right)$. The advantage of the adaptive protocol is that, it can automatically scale down the transmission probability for an initial $\tau_{0}$ that is overestimated. Hence, as shown in Figs. 3 and 5, the adaptive protocol is more robust in the region where $\tau_{0}>1 / K$. We note that for small $\tau_{0}<1 / K$, in which case $\tau_{0}$ is underestimated, the nonadaptive protocol requires a slightly smaller $N_{s}\left(\tau_{0}\right)$ than that of the adaptive protocol. This is because the adaptive protocol decreases $\tau_{0}$ after each collision, which decreases the number of transmitted BMs further and hence yields a slight performance loss compared to the nonadaptive protocol.

\section{CONCLUSION}

In this paper, we presented random access protocols for exchange of sensing information among SUs for collaborative spectrum sensing in cognitive radio networks. Both adaptive and nonadaptive protocols were considered. A general mathematical framework that allows analysis of the proposed protocols under very broad conditions was developed and its perfect accuracy was corroborated through computer simulations. Such analysis characterizes the detection reliability achieved through collaborative sensing, and is used to determine the optimal protocol parameters and the length of the signaling period for a cognitive radio network. The analysis developed allows consideration of hidden nodes and the cases where each SU node senses only a subset of PU bands. We believe that this is the first work that thoroughly analyzes the random access protocols when applied to signaling for collaborative spectrum sensing in cognitive radio networks.

We note that the random access protocols presented in this paper can be generalized in a number of ways. For instance, besides "multiplicative decrease," we can also adopt "additive increase" so that the transmission probability $\tau$ can be increased when the signaling traffic is low. Also, the proposed protocols are applicable to the more realistic scenario when the communication within the SU network is imperfect, i.e., an SU might not be able to receive the SBM correctly even when there is no collision, or an SU might not be able to detect a collision when it occurs. Another issue that warrants further study is the probability of false alarm, which is caused by the broadcast of PU activities that are actually absent. This issue can be addressed by introducing some detection/decision mechanisms based on a set of SBMs that an SU receives. Extensions of the theoretical analysis to these more sophisticate protocols, however, are nontrivial and deserve further investigation.

\section{APPENDIX A}

PROOF OF (5) AND (6)

Let $\mathbf{s}=\left\{s_{1}, \cdots, s_{B}\right\} \subset\left\{t_{1}, t_{2}, \cdots, t_{C}\right\}$ denote the $B$ PU bands that an SU chooses to sense. First, we write

$$
\begin{aligned}
P\left(X_{c_{1}, c}\right) & =\sum_{i=1}^{d} r_{i} P\left(X_{c_{1}, c} \mid q_{i}\right) \\
& =\sum_{i=1}^{d} r_{i} \sum_{\mathbf{s}} P(\mathbf{s}) P\left(X_{c_{1}, c} \mid q_{i}, \mathbf{s}\right) \\
& =\frac{1}{\left(\begin{array}{c}
C \\
B
\end{array}\right)} \sum_{i=1}^{d} r_{i} \sum_{\mathbf{s}} P\left(X_{c_{1}, c} \mid q_{i}, \mathbf{s}\right) .
\end{aligned}
$$

Consider $I_{\mathbf{s}}=\left\{s_{1}, \cdots, s_{B}\right\} \bigcap\left\{t_{1}, t_{2}, \cdots, t_{c}\right\}$. Let $j=\left|I_{\mathbf{s}}\right|$ denote the number of elements in $I_{\mathrm{s}}$. Clearly, we must have $j \leq \min (B, c)$, which gives the upper limit of the summation in (5). For a given $j$, we examine all choices of $\mathbf{s}$ such that the term $P\left(X_{c_{1}, c} \mid q_{i}, \mathbf{s}\right)$ in (28) is greater than zero. In order for $P\left(X_{c_{1}, c} \mid q_{i}, \mathbf{s}\right)>0$, we must have $\left\{t_{1}, t_{2}, \cdots, t_{c_{1}}\right\} \subset\left\{s_{1}, \cdots, s_{B}\right\}$ and thus the SU must sense $j-c_{1}$ PU bands out of PU bands $\left\{t_{c_{1}+1}, \cdots, t_{c}\right\}$, and he must also sense $B-j$ PU bands out of PU bands $\left\{t_{c+1}, \cdots, t_{C}\right\}$. It follows that $j \geq c_{1}$ and $j \geq B-(C-c)$. These specify the lower limit of the summation in (5). Hence, the total number of $\mathbf{s}$ is given by $\left(\begin{array}{c}c-c_{1} \\ j-c_{1}\end{array}\right)\left(\begin{array}{l}C-c \\ B-j\end{array}\right)$. For each of such $\mathbf{s}$, we have $P\left(X_{c_{1}, c} \mid q_{i}, \mathbf{s}\right)=q_{i}^{c_{1}}\left(1-q_{i}\right)^{j-c_{1}}$ because with probability $q_{i}$, 
the SU detects each of the PU bands $\left\{t_{1}, t_{2}, \cdots, t_{c_{1}}\right\}$, and with probability $1-q_{i}$ the $\mathrm{SU}$ fails to detect any of the $j-c_{1}$ PU bands in $I_{\mathbf{S}}-\left\{t_{1}, t_{2}, \cdots, t_{c_{1}}\right\}$. The detection results of the $\mathrm{SU}$ for any of the PU bands in $\left\{t_{1}, t_{2}, \cdots, t_{C}\right\}-\left\{t_{1}, t_{2}, \cdots, t_{c}\right\}$ becomes irrelevant, and hence they do not appear in (5).

Similar to (28), we write $P\left(Y_{c_{1}}\right)$ as

$$
P\left(Y_{c_{1}}\right)=\frac{1}{\left(\begin{array}{l}
C \\
B
\end{array}\right)} \sum_{i=1}^{d} r_{i} \sum_{\mathbf{s}} P\left(Y_{c_{1}} \mid q_{i}, \mathbf{s}\right) .
$$

We define $I_{\mathbf{s}}=\left\{s_{1}, \cdots, s_{B}\right\} \bigcap\left\{t_{1}, t_{2}, \cdots, t_{c_{1}}\right\}$ and let $j=\left|I_{\mathbf{s}}\right|$ denote the number of elements in $I_{\mathrm{s}}$. For a given $j$, we examine all possible $\mathbf{s}$ such that $P\left(Y_{c_{1}} \mid q_{i}, \mathbf{s}\right)>0$. The total number of such s equals $\left(\begin{array}{c}c_{1} \\ j\end{array}\right)\left(\begin{array}{c}C-c_{1} \\ B-j\end{array}\right)$, and $P\left(Y_{c_{1}} \mid q_{i}, \mathbf{s}\right)=\left(1-q_{i}\right)^{j}$.

\section{APPENDIX B}

PROOF OF PROPOSITION 4.1

First, we have (30), shown as follows:

$$
\begin{aligned}
& P\left(B_{c, n}\right)= P\left(\bigcup_{k=1}^{K} B_{c, n} D_{k}\right)=\sum_{k=1}^{K} P\left(B_{c, n} D_{k}\right) \\
&= \sum_{k=1}^{K} P\left(D_{k}\right) P\left(B_{c, n} \mid D_{k}\right) \\
&= \sum_{k=1}^{K} P\left(D_{k}\right)\left(\sum_{n_{1}=1}^{n-1} P\left(F_{n_{1}} \mid D_{k}\right) P\left(B_{c, n} \mid D_{k}, F_{n_{1}}\right)\right. \\
&\left.\quad+P\left(F_{n} \mid D_{k}\right) P\left(B_{c, n} \mid D_{k}, F_{n}\right)\right) \\
&=\sum_{k=1}^{K} P\left(D_{k}\right)\left(\sum_{n_{1}=1}^{n-1} P\left(F_{n_{1}} \mid D_{k}\right) P\left(B_{c, n} \mid D_{k}, F_{n_{1}}\right)\right) \\
&+\sum_{k=1}^{K-1}\left(P\left(D_{k}\right) P\left(F_{n} \mid D_{k}\right) P\left(B_{c, n} \mid D_{k}, F_{n}\right)\right) \\
&+P\left(D_{K}\right) P\left(F_{n} \mid D_{K}\right) P\left(B_{c, n} \mid D_{K}, F_{n}\right) .
\end{aligned}
$$

Conditioned upon $F_{n}$, we note that for $B_{c, n}$ to occur, all $c$ busy PU bands should be reported by SBM1. Hence, we have

$$
P\left(B_{c, n} \mid D_{k}, F_{n}\right)=P\left(G_{c, c}\right) \quad \text { for every } 1 \leq k \leq K-1 .
$$

Therefore, to prove (10), it is sufficient to show that

$P\left(D_{K}\right) P\left(B_{c, n} \mid D_{K}, F_{n}\right)=P\left(D_{K} U\right)-P\left(D_{K}\right)\left(1-P\left(G_{c, c}\right)\right)$.

To prove (32), we let $\bar{U}$ denote the complement of the event $U$ and note that

$$
\begin{aligned}
P\left(B_{c, n} \mid D_{K}, F_{n}\right)= & P\left(U \mid D_{K}, F_{n}\right) P\left(B_{c, n} \mid D_{K}, F_{n}, U\right) \\
& +P\left(\bar{U} \mid D_{K}, F_{n}\right) P\left(B_{c, n} \mid D_{K}, F_{n}, \bar{U}\right) \\
= & P\left(U \mid D_{K}\right) P\left(G_{c, c} \mid D_{K}, U\right)
\end{aligned}
$$

where the second line follows since 1) $P\left(U \mid D_{K}, F_{n}\right)=$ $P\left(U \mid D_{K}\right)$ because $U$ is independent of $F_{n}$. 2) $P\left(B_{c, n} \mid D_{K}, F_{n}, U\right)=P\left(G_{c, c} \mid D_{K}, U\right)$ because conditioned upon $F_{n}$ and $U$, in order for $B_{c, n}$ to occur, the SBM1 must contain all $c$ busy PU bands. 3) $P\left(B_{c, n} \mid D_{K}, F_{n}, \bar{U}\right)=0$. Moreover, since

$$
\begin{aligned}
P\left(G_{c_{1}, c} \mid D_{K}, U\right) & =\frac{P\left(G_{c_{1}, c} D_{K} U\right)}{P\left(D_{K} U\right)} \\
& =\frac{P\left(D_{K}\right) P\left(G_{c_{1}, c} U \mid D_{K}\right)}{P\left(D_{K} U\right)} \\
& =\frac{P\left(D_{K}\right) P\left(G_{c_{1}, c}\right)}{P\left(D_{K} U\right)}
\end{aligned}
$$

we have

$$
\begin{aligned}
P\left(G_{c, c} \mid D_{K}, U\right) & =1-\sum_{c_{1}=1}^{c-1} P\left(G_{c_{1}, c} \mid D_{K}, U\right) \\
& =1-\frac{P\left(D_{K}\right)}{P\left(D_{K} U\right)}\left(1-P\left(G_{c, c}\right)\right) .
\end{aligned}
$$

It then follows from (33) and (35) that

$$
\begin{aligned}
P & \left(D_{K}\right) P\left(B_{c, n} \mid D_{K}, F_{n}\right) \\
& =P\left(D_{K}\right) P\left(U \mid D_{K}\right) P\left(G_{c, c} \mid D_{K}, U\right) \\
& =P\left(D_{K} U\right)\left[1-\frac{P\left(D_{K}\right)}{P\left(D_{K} U\right)}\left(1-P\left(G_{c, c}\right)\right)\right] \\
& =P\left(D_{K} U\right)-P\left(D_{K}\right)\left(1-P\left(G_{c, c}\right)\right) .
\end{aligned}
$$

APPENDIX C

PROOF OF PROPOSITION 4.3

Using the law of total probability, we obtain (37) shown as follows:

$$
\begin{aligned}
P( & \left.B_{c, n} \mid D_{k}, F_{n_{1}}\right) \\
= & \sum_{c_{1}=1}^{c-1} \sum_{k_{1}=1}^{k-1} P\left(G_{c_{1}, c} H_{k_{1}} \mid D_{k}, F_{n_{1}}\right) \\
& \times P\left(B_{c, n} \mid D_{k}, F_{n_{1}}, G_{c_{1}, c}, H_{k_{1}}\right) \\
= & \sum_{c_{1}=1}^{c-1} \sum_{k_{1}=1}^{k-1} P\left(G_{c_{1}, c} \mid D_{k}, F_{n_{1}}\right) P\left(H_{k_{1}} \mid D_{k}, F_{n_{1}}, G_{c_{1}, c}\right) \\
& \times P\left(B_{c, n} \mid D_{k}, F_{n_{1}}, G_{c_{1}, c}, H_{k_{1}}\right) \\
= & \sum_{c_{1}=1}^{c-1} \sum_{k_{1}=1}^{k-1} P\left(G_{c_{1}, c}\right) P\left(H_{k_{1}} \mid D_{k}, G_{c_{1}, c}\right) \\
& \times P\left(B_{c-c_{1}, n-n_{1}} \mid \tilde{D}_{k_{1}}\right) .
\end{aligned}
$$

The second line of (37) follows from the Bayes' rule and the third line follows from 1) $P\left(G_{c_{1}, c} \mid D_{k}, F_{n_{1}}\right)=P\left(G_{c_{1}, c}\right)$ since the number of the reported busy PU bands by an active user does not depend on when SBM1 has been transmitted and how many active users existed; 2) $P\left(H_{k_{1}} \mid D_{k}, F_{n_{1}}, G_{c_{1}, c}\right)=P\left(H_{k_{1}} \mid D_{k}, G_{c_{1}, c}\right)$ since the number of the remaining active users does not depend on when SBM1 has been transmitted; and 3) the identity $P\left(B_{c, n} \mid D_{k}, F_{n_{1}}, G_{c_{1}, c}, H_{k_{1}}\right)=P\left(B_{c-c_{1}, n-n_{1}} \mid \tilde{D}_{k_{1}}\right)$ follows since after SBM1, given the values of $n_{1}, c_{1}$, and $k_{1}$, the signaling continues with $c-c_{1}$ busy PU bands to be broadcasted, $n-n_{1}$ time slots remaining before reaching the time slot $n$, 
and $k_{1}$ active users with new information to broadcast plus the dummy user that transmitted SBM1.

To develop (17), we first note that for every $1 \leq k \leq K-1$, we have

$$
\begin{array}{r}
P\left(B_{c, n} \mid \tilde{D}_{k}\right)=\sum_{n_{1}=1}^{n-1} P\left(F_{n_{1}} \mid \tilde{D}_{k}\right) \\
P\left(B_{c, n} \mid \tilde{D}_{k}, F_{n_{1}}\right) \\
+P\left(F_{n} \mid \tilde{D}_{k}\right) P\left(G_{c, c}\right) .
\end{array}
$$

Next, we have

$$
\begin{aligned}
P\left(B_{c, n} \mid \tilde{D}_{k}, F_{n_{1}}\right)= & P\left(R \mid \tilde{D}_{k}, F_{n_{1}}\right) P\left(B_{c, n} \mid \tilde{D}_{k}, F_{n_{1}}, R\right) \\
& +P\left(\bar{R} \mid \tilde{D}_{k}, F_{n_{1}}\right) P\left(B_{c, n} \mid \tilde{D}_{k}, F_{n_{1}}, \bar{R}\right) \\
= & \frac{1}{k+1} P\left(B_{c, n-n_{1}} \mid \tilde{D}_{k}\right) \\
& +\frac{k}{k+1} P\left(B_{c, n} \mid D_{k}, F_{n_{1}}\right) .
\end{aligned}
$$

In writing the second line of (39), we note that 1) the identities $P\left(R \mid \tilde{D}_{k}, F_{n_{1}}\right)=1 /(k+1)$ and $P\left(\bar{R} \mid \tilde{D}_{k}, F_{n_{1}}\right)=k /(k+1)$ follow since we assume that the dummy user and the regular active users transmit BMs with the same probability $\left.\tau_{0} ; 2\right) P\left(B_{c, n} \mid \tilde{D}_{k}, F_{n_{1}}, R\right)=P\left(B_{c, n-n_{1}} \mid \tilde{D}_{k}\right)$ since when the dummy user sends an SBM in time slot $n_{1}$, the only change in the state of network is that the remaining time slots left for signaling becomes $n-n_{1}$; and 3) $P\left(B_{c, n} \mid \tilde{D}_{k}, F_{n_{1}}, \bar{R}\right)=P\left(B_{c, n} \mid D_{k}, F_{n_{1}}\right)$ since assuming that SBM1 was transmitted by a regular user, the presence of the dummy user becomes irrelevant to the behavior of the network. This is because upon receiving the SBM, the dummy user stops transmission as this SBM contains the information that is carried by the dummy user. The regular user who just sent the SBM will now become the dummy user. We can then substitute (39) in (38) to obtain (17).

\section{APPENDIX D}

\section{PROOF OF PROPOSITION 4.2}

Let us define the events

$$
\begin{aligned}
& Z=\{\mathrm{An} \mathrm{SU} \text { is active at the end of the sensing } \\
& \text { period } \\
& W_{c_{1}, c}=\{\mathrm{By} \text { the end of the sensing period an } \mathrm{SU} \\
& \text { in detects } \left.c_{1} \text { out of } c \text { busy PU bands }\right\} \\
& W_{1}=\{\text { An SU detects at least one busy PU band } \\
& \text { out of } c-c_{1} \text { busy PU bands }
\end{aligned}
$$

Using these definitions, one finds that

$$
\begin{aligned}
P\left(D_{k}\right) & =\left(\begin{array}{c}
K \\
k
\end{array}\right)[P(Z)]^{k}[1-P(Z)]^{K-k} \\
P\left(D_{K} U\right) & =P\left(D_{K}\right)-P\left(D_{K} \bar{U}\right)=P\left(D_{K}\right)-P(\bar{U}) \\
P\left(G_{c_{1}, c}\right) & =P\left(W_{c_{1}, c} \mid Z\right)=\frac{P\left(W_{c_{1}, c} Z\right)}{P(Z)} \\
& =\frac{P\left(W_{c_{1}, c}\right)}{P(Z)}
\end{aligned}
$$

and

$$
P\left(Q_{c_{1}, c}\right)=P\left(W_{1} \mid Z\right)=\frac{P\left(W_{1} Z\right)}{P(Z)}=\frac{P\left(W_{1}\right)}{P(Z)} .
$$

Noting that $P(Z)=1-P\left(Y_{c}\right), P\left(D_{K}\right)=[P(Z)]^{K}$, $P(\bar{U})=\left[P\left(X_{c, c}\right)\right]^{K}, P\left(W_{c_{1}, c}\right)=\left(\begin{array}{c}c \\ c_{1}\end{array}\right) P\left(X_{c_{1}, c}\right)$, and $P\left(W_{1}\right)=1-P\left(Y_{c-c_{1}}\right)$, the above results can be written in the forms (12)-(15).

\section{APPENDIX E}

PROOF OF PROPOSITION 5.2

Similar to (37), we have

$$
\begin{aligned}
P\left(B_{c, n} \mid D_{k}, F_{n_{1}, z_{1}}\right)=\sum_{c_{1}=1}^{c-1} & \sum_{k_{1}=1}^{k-1} P\left(G_{c_{1}, c}\right) P\left(H_{k_{1}} \mid D_{k}, G_{c_{1}, c}\right) \\
& \times P\left(B_{c-c_{1}, n-n_{1}} \mid \hat{D}_{k_{1}, z_{1}+1}\right)
\end{aligned}
$$

Next, similar to (38), we have for every $1 \leq k \leq K-1$

$$
\begin{aligned}
P\left(B_{c, n} \mid \hat{D}_{k, z_{d}}\right)= & \sum_{n_{1}=1}^{n-1} \sum_{z_{1}=0}^{n_{1}-1} P\left(F_{n_{1}, z_{1}} \mid \hat{D}_{k, z_{d}}\right) \\
& \times P\left(B_{c, n} \mid \hat{D}_{k, z_{d}}, F_{n_{1}, z_{1}}\right) \\
& +\sum_{z_{1}=0}^{n-1} P\left(F_{n, z_{1}} \mid \hat{D}_{k, z_{d}}\right) P\left(G_{c, c}\right) .
\end{aligned}
$$

The dual of (39) is written as

$$
\begin{aligned}
P( & \left.B_{c, n} \mid \hat{D}_{k, z_{d}}, F_{n_{1}, z_{1}}\right) \\
= & P\left(R \mid \hat{D}_{k, z_{d}}, F_{n_{1}, z_{1}}\right) P\left(B_{c, n} \mid \hat{D}_{k, z_{d}}, F_{n_{1}, z_{1}}, R\right) \\
& +P\left(\bar{R} \mid \hat{D}_{k, z_{d}}, F_{n_{1}, z_{1}}\right) P\left(B_{c, n} \mid \hat{D}_{k, z_{d}}, F_{n_{1}, z_{1}}, \bar{R}\right) \\
= & P\left(R \mid \hat{D}_{k, z_{d}}, F_{n_{1}, z_{1}}\right) P\left(B_{c, n-n_{1}} \mid \hat{D}_{k, z_{d}+z_{1}+1}\right) \\
& +P\left(\bar{R} \mid \hat{D}_{k, z_{d}}, F_{n_{1}, z_{1}}\right) P\left(B_{c, n} \mid D_{k}, F_{n_{1}, z_{1}}\right)
\end{aligned}
$$

Conditioned upon $\hat{D}_{k, z_{d}}$ and $F_{n_{1}, z_{1}}$, the transmission probability of the dummy user during time slot $n_{1}$ must be $\tau_{0} \alpha^{z_{d}+z_{1}}$ and the transmission probability of a regular user must be $\tau_{0} \alpha^{z_{1}}$. Hence, we obtain (47) as follows:

$$
\begin{aligned}
P & \left(R \mid \hat{D}_{k, z_{d}}, F_{n_{1}, z_{1}}\right) \\
& =\frac{\left[1-\tau_{0} \alpha^{z_{1}}\right]^{k}\left(\tau_{0} \alpha^{z_{d}+z_{1}}\right)}{\left[1-\tau_{0} \alpha^{z_{1}}\right]^{k}\left(\tau_{0} \alpha^{z_{d}+z_{1}}\right)+k\left[1-\tau_{0} \alpha^{z_{1}}\right]^{(k-1)}\left(\tau_{0} \alpha^{z_{1}}\right)\left(1-\tau_{0} \alpha^{\left.z_{d}+z_{1}\right)}\right.} \\
& =\frac{\left(1-\tau_{0} \alpha^{z_{1}}\right) \alpha^{z_{d}}}{k+\alpha^{z_{d}}-(k+1) \tau_{0} \alpha^{z_{d}+z_{1}}} .
\end{aligned}
$$

\section{REFERENCES}

[1] S. Haykin, "Cognitive radio: Brain-empowered wireless communications," IEEE J. Sel. Areas Commun., vol. 23, no. 2, pp. 201-220, Feb. 2005.

[2] A. S. Tanenbaum, Computer Networks. Englewood Cliffs, NJ: Prentice-Hall, 1989.

[3] B. Farhang-Boroujeny, "Filter bank spectrum sensing for cognitive radios," IEEE Trans. Signal Process., vol. 56, no. 5, pp. 1801-1811, May 2008.

[4] T. Weiss, A. Krohn, J. Hillenbrand, and F. Jondral, "Efficient signaling of spectral resources in spectrum pooling systems," in Proc. 10th Symp. Commun. Veh.Technol. (SCVT), Nov. 2003 [Online]. Available: http:/ www.teco.edu/krohn/efficient_signaling.pdf

[5] C. Han, J. Wang, and S. Li, "A spectrum exchange mechanism in cognitive radio contexts," in Proc. IEEE 17th Int. Symp. Personal, Indoor, Mobile Radio Commun., Sep. 2006, pp. 1-5. 
[6] E. Visotsky, S. Kuffner, and R. Peterson, "On collaborative detection of TV transmissions in support of dynamic spectrum sharing," in Proc. 1st IEEE Int. Symp. New Frontiers Dynamic Spectrum Access Networks (DySPAN), Nov. 2005, pp. 338-345.

[7] H. Su and X. Zhang, "Cognitive radio based multi-channel mac protocols for wireless ad hoc networks," in Proc. IEEE Global Telecomm. Conf. (GLOBECOM), Nov. 2007, pp. 4857-4861.

[8] D. Bertsekas and R. Gallenger, Data Networks. Englewood Cliffs, NJ: Prentice-Hall, 1992.

[9] E. Azarnasab, R. Kempter, N. Patwari, and B. Farhang-Boroujeny, "Filterbank multicarrier and multicarrier cdma for cognitive radio systems," in Proc. IEEE CrownCom, Aug. 2007, pp. 472-481.

[10] D. Cabric, S. Mishra, and R. Brodersen, "Implementation issues in spectrum sensing for cognitive radios," in Conf. Rec. 38th Asilomar Conf. Signals, Syst., Comput., Nov. 2004, vol. 1, pp. 772-776.

[11] S. Mishra, A. Sahai, and R. Brodersen, "Cooperative sensing among cognitive radios," in Proc. IEEE Int. Conf. Commun. (ICC), Jun. 2006, vol. 4, pp. $1658-1663$.

[12] D. Cabric, S. M. Mishra, D. Willkomm, R. Brodersen, and A. Wolisz, "A cognitive radio approach for usage of virtual unlicensed spectrum," in Proc. 14th IST Mobile and Wireless Commun. Summit, Jun. 2005.

[13] H. Nan, T.-I. Hyon, and S.-J. Yoo, "Distributed coordinated spectrum sharing mac protocol for cognitive radio," in Proc. IEEE Int. Symp. New Frontiers in Dynamic Spectrum Access Networks (DySPAN), Apr. 2007, pp. 240-249.

[14] T. Weiss and F. Jondral, "Spectrum pooling: And innovative strategy for the enhancement of spectrum efficiency," IEEE Commun. Mag., vol. 42, no. 3, pp. S8-S14, Mar. 2004.

[15] B. Hamdaoui and K. Shin, "OS-Mac: An efficient Mac protocol for spectrum-agile wireless networks," IEEE Trans. Mobile Comput., vol. 7, no. 8, pp. 915-930, Aug. 2008.

[16] J. Jia, Q. Zhang, and X. Shen, "HC-Mac: A hardware-constrained cognitive Mac for efficient spectrum management," IEEE J. Sel. Areas Commun., vol. 26, no. 1, pp. 106-117, Jan. 2008.

[17] C. Cordeiro and K. Challapali, "C-mac: A cognitive mac protocol for multi-channel wireless networks," in Proc. IEEE Int. Symp. New Frontiers in Dynamic Spectrum Access Networks (DySPAN), Apr. 2007, pp. $147-157$.

[18] H. Su and X. Zhang, "Opportunistic mac protocols for cognitive radio based wireless networks," in Proc., 41st Annu. Conf. Inf. Sci. Syst. (CISS), Mar. 2007, pp. 363-368.

[19] Q. Zhao, L. Tong, and A. Swami, "Decentralized cognitive mac for dynamic spectrum access," in Proc. 1st IEEE Int. Symp. New Frontiers in Dynamic Spectrum Access Networks (DySPAN), Nov. 2005, pp. 224-232.

[20] D. Djonin, Q. Zhao, and V. Krishnamurthy, "Optimality and complexity of opportunistic spectrum access: A truncated Markov decision process formulation," in Proc. IEEE Int. Conf. Commun. (ICC), Jun. 2007, pp. 5787-5792.

[21] K. T. E. Azarnasab, R.-R. Chen, and Z. Tao, "Medium access control signaling for reliable spectrum agile radios," in Proc. IEEE Golbecom, Nov. 2009.

[22] F. C. Commission, Spectrum Policy Task Force, ET Docket 02-135, Nov. 2002.

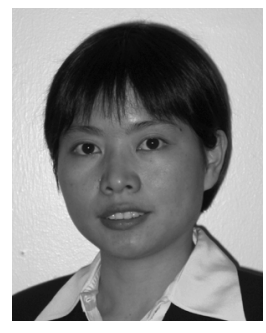

Rong-Rong Chen received the B.Sc. degree in applied mathematics from TsingHua University, Beijing, China, in 1994 and the M.Sc. degree in mathematics and the Ph.D. degree in electrical and computer engineering from the University of Illinois at Urbana-Champaign (UIUC), in 1996 and 2003, respectively.

Since August 2003, She has been an Assistant Professor in the Department of Electrical and Computer Engineering, University of Utah, Salt Lake City. Her main research interest is in communication systems and networks, with current emphasis on MIMO communication, statistical soft detection, underwater acoustic communication, and cognitive radio networks.

Dr. Chen was the recipient of the University Fellowship for graduate study in the mathematics department at UIUC and the M. E. Van Valkenburg Graduate Research Award for excellence in doctoral research in the Department of Electrical and Computer Engineering at UIUC. She is a recipient of the prestigious National Science Foundation (NSF) Faculty Early Career Development (CAREER) Award in 2006.

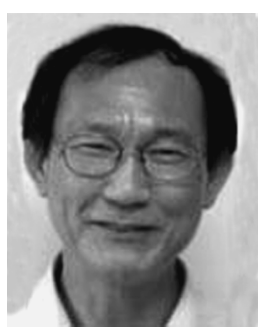

Koon Hoo Teo received the M.Sc. and Ph.D. degrees from University of Alberta, Edmonton, AB, Canada, in 1985 and 1990, respectively.

He was with Nortel for about 15 years where he has worked in wireless system research and product development. For the last five years at Nortel, he worked as a Radio Architect and a System Engineering Manager where he was actively involved in the research and implementation issues of a number of $3 \mathrm{G}$ and $4 \mathrm{G}$ wireless systems and translated a number of initial research concepts into highly competitive products. The wireless systems he worked in include Wireless Mesh Networks and WiMAX systems. He left Nortel and joined Mitsubishi Electric Research Laboratories (MERL), Cambridge, MA, in 2006. His work at MERL includes cognitive radio, game theory, wireless mesh and multi-hop systems. His current research interest includes theory, simulation, and characterization of meta material and its applications in the energy and communication space. He is currently a holder of more than 20 U.S. patents and at least another 35 pending. He is also the author and coauthor of more than 35 journal and conference papers.

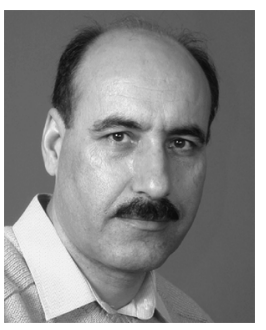

Behrouz Farhang-Boroujeny (M'84-SM'90) received the B.Sc. degree in electrical engineering from Teheran University, Tehran, Iran, in 1976, the M.Eng. degree from University of Wales Institute of Science and Technology, Cardiff, U.K., in 1977, and the Ph.D. degree from Imperial College, University of London, London, U.K., in 1981.

From 1981 to 1989 , he was with the Isfahan University of Technology, Isfahan, Iran. From 1989 to 2000, he was with the National University of Singapore. Since August 2000, he has been with the University of Utah, Salt Lake City, where he is now a Professor and Associate Chair of the department. He is an expert in the general area of signal processing. His current scientific interests are adaptive filters, multicarrier communications, detection techniques for space-time coded systems, cognitive radio, and signal processing applications to optical devices. In the past, he has worked and has made significant contribution to areas of adaptive filters theory, acoustic echo cancellation, magnetic/optical recoding, and digital subscriber line technologies. He is the author of the books Adaptive Filters: Theory and applications (Wiley, 1998) and Signal Processing Techniques for Software Radios, self published at Lulu publishing house, 2009.

Dr. Farhang-Boroujeny received the UNESCO Regional Office of Science and Technology for South and Central Asia Young Scientists Award in 1987. $\mathrm{He}$ served as an Associate Editor of the IEEE TRAnSACtions on Signal PROCESSING from July 2002 to July 2005. He is now serving as an Associate Editor of the IEEE SignAl PROCESSING LETTERS. He has also been involved in various IEEE activities, including the chairmanship of the Signal Processing/Communications chapter of IEEE of Utah in 2004 and 2005. 